\title{
Scaling of normalized mean energy and scalar dissipation rates in a turbulent channel flow
}

\author{
Hiroyuki Abe ${ }^{1, a)}$ and Robert Anthony Antonia 2, b) \\ ${ }^{1}$ Japan Aerospace Exploration Agency, Tokyo 182-8522, Japan \\ ${ }^{2}$ Discipline of Mechanical Engineering, University of Newcastle, Newcastle, New South Wales 2308, Australia
}

(Received 26 July 2010; accepted 6 April 2011; published online 25 May 2011)

\begin{abstract}
Non-dimensional parameters for the mean energy and scalar dissipation rates $C_{\varepsilon}$ and $C_{\varepsilon \theta}$ are examined using direct numerical simulation (DNS) data obtained in a fully developed turbulent channel flow with a passive scalar $(P r=0.71)$ at several values of the Kármán (Reynolds) number $h^{+}$. It is shown that $C_{\varepsilon}$ and $C_{\varepsilon \theta}$ are approximately equal in the near-equilibrium region (viz., $y^{+}=100$ to $y / h=0.7$ ) where the production and dissipation rates of either the turbulent kinetic energy or scalar variance are approximately equal and the magnitudes of the diffusion terms are negligibly small. The magnitudes of $C_{\varepsilon}$ and $C_{\varepsilon \theta}$ are about 2 and 1 in the logarithmic and outer regions, respectively, when $h^{+}$is sufficiently large. The former value is about the same for the channel, pipe, and turbulent boundary layer, reflecting the similarity between the mean velocity and temperature distributions among these three canonical flows. The latter value is, on the other hand, about twice as large as in homogeneous isotropic turbulence due to the existence of the large-scale $u$ structures in the channel. The behaviour of $C_{\varepsilon}$ and $C_{\varepsilon \theta}$ impacts on turbulence modeling. In particular, the similarity between $C_{\varepsilon}$ and $C_{\varepsilon \theta}$ leads to a simple relation for the scalar variance to turbulent kinetic energy time-scale ratio, an important ingredient in the eddy diffusivity model. This similarity also yields a relation between the Taylor and Corrsin microscales and analogous relations, in terms of $h^{+}$, for the Taylor microscale Reynolds number and Corrsin microscale Peclet number. This dependence is reasonably well supported by both the DNS data at small to moderate $h^{+}$and the experimental data of Comte-Bellot [Ph. D. thesis (University of Grenoble, 1963)] at larger $h^{+}$. It does not however apply to a turbulent boundary layer where the mean energy dissipation rate, normalized on either wall or outer variables, is about $30 \%$ larger than for the channel flow. (C) 2011 American Institute of Physics. [doi:10.1063/1.3584124]
\end{abstract}

\section{INTRODUCTION}

Over the past several decades, significant attention has been given to the mean energy dissipation rate,

$$
\bar{\varepsilon}=v \overline{u_{i, j}\left(u_{i, j}+u_{j, i}\right)},
$$

where $u_{1}, u_{2}$, and $u_{3}$ denote the streamwise, wall-normal, and spanwise velocity fluctuations, respectively; $u, v, w$ are sometimes used interchangeably with $u_{1}, u_{2}, u_{3} ; v$ denotes the kinematic viscosity and the overbar denotes averaging with respect to $x, z$ ( $x, y, z$ denote the streamwise, wall-normal, and spanwise directions, respectively) and $t$ (time), of the turbulent kinetic energy $k\left(\equiv \overline{u_{i}^{2}} / 2\right)$ owing to the dissipative nature of turbulence (see, for example, the pioneering work of Taylor ${ }^{1}$ and Kolmogorov ${ }^{2}$ and, more recently, the review of Sreenivasan and Antonia $\left.^{3}\right) . \bar{\varepsilon}$ represents the rate at which the energy is dissipated at the molecular level. In statistically steady turbulence, this magnitude is equivalent to the rate of energy transfer from large to small scales in the energy cascade where it is classically assumed that eddies break up successively through inertial forces until their size becomes comparable to the Kolmogorov length scale

\footnotetext{
${ }^{a)}$ Electronic mail: habe@chofu.jaxa.jp.

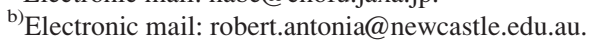

$\eta\left(\equiv\left(v^{3} / \bar{\varepsilon}\right)^{1 / 4}\right)$. This has led to the idea that $\bar{\varepsilon}$ should become independent of the viscosity in the limit of infinite Reynolds number, thus suggesting a non-dimensional parameter

$$
C_{\varepsilon}=\bar{\varepsilon} \ell / v^{3}
$$

where $v$ and $\ell$ denote the energy containing scales of velocity and length, respectively. This form was first addressed by Taylor; ${ }^{1}$ it is sometimes referred to as the zeroth law of turbulence (e.g., Pearson et $\mathrm{l}^{4}$ ); it is interpreted as the ratio of the kinetic energy $v^{2}$ to the corresponding time scale $\ell / v$. To date, the possibility that the magnitude of $C_{\varepsilon}$ becomes constant has been argued extensively in the literature (e.g., Batchelor, ${ }^{5}$ Sreenivasan, ${ }^{6-8}$ Lumley, ${ }^{9}$ Frisch, ${ }^{10}$ Kaneda et al.,${ }^{11}$ Antonia et al..${ }^{12}$ Antonia and Pearson, ${ }^{13}$ Burattini et al. ${ }^{14}$ and Goto and Vassilicos ${ }^{15}$ ). The constancy is also associated with the Re independence of the drag coefficient (Frisch ${ }^{10}$ ) and other turbulence modeling parameters, as mentioned below.

Following Eq. (2), the non-dimensional parameter,

$$
C_{\varepsilon}=\bar{\varepsilon} L_{u u} / u^{13},
$$

has been examined in detail in the wake of the seminal experimental work of Batchelor, ${ }^{5}$ where $\ell=L_{u u}$ and $v=u^{\prime}$ ( $L_{u u}$ is the integral length scale of $u$ and the prime denotes 
the root-mean-square value). Unless otherwise stated, $L_{u и}$ is hereafter defined as

$$
L_{u u}=\int_{0}^{\infty} R_{u u}(r) d r,
$$

where $R_{u u}(r)\left(\equiv \overline{u(x) u(x+r)} / u^{\prime 2}\right)$ is the longitudinal twopoint correlation of $u$. Sreenivasan ${ }^{7}$ collected a large body of experimental data for Eq. (3) in grid turbulence and pointed out that $C_{\varepsilon}$ becomes constant when the Taylor microscale Reynolds number $R_{\lambda 1}\left(\equiv u^{\prime} \lambda_{1} / v\right)$ is larger than $50\left(\lambda_{1}\left(\equiv u^{\prime}\right)\right.$ $\left.u,{ }_{1}^{\prime}\right)$ is the longitudinal Taylor microscale). Sreenivasan ${ }^{7}$ also evaluated $C_{\varepsilon}$ in other turbulent flows, observing that the effects of shear and inhomogeneity are not negligible, viz., the magnitude of $C_{\varepsilon}$ in either homogenous shear flows or inhomogeneous flows is not the same as in grid turbulence. In the latter context, Burattini et al. ${ }^{14}$ revisited the magnitude of $C_{\varepsilon}$ in grid turbulence, two-dimensional wakes, and homogeneous shear flows. They noted that it exhibits a significant variation, typically in the range $0.5-2.5$, for $R_{\lambda 1}>50$. The previous studies suggest that the magnitude of $C_{\varepsilon}$ becomes constant when $R_{\lambda 1}$ is sufficiently large but that it depends on the type of flow. Even in the same flow, there is evidence to suggest that the magnitude can depend strongly on the initial conditions (e.g., Antonia et al. ${ }^{12}$ and Antonia and Pearson ${ }^{13}$ ).

A significant amount of data for $C_{\varepsilon}$ has also been obtained from direct numerical simulations (DNSs) of homogeneous isotropic turbulence. In the latter flow, $L_{u u}$ is defined as

$$
L_{u u}=\frac{\pi}{2 u^{\prime 2}} \int_{0}^{\infty} \frac{E(k)}{k} d k,
$$

where $E(k)$ is the 3D energy spectrum and $k$ the 3D wavenumber. From his survey of existing DNS data $\left(R_{\lambda 1}=21-240\right)$, Sreenivasan ${ }^{8}$ indicated that while $C_{\varepsilon}$ tends to become constant for $R_{\lambda 1}>100$, its magnitude depends on how the turbulence is forced at low wavenumbers. Goto and Vassilicos ${ }^{15}$ varied this large-scale forcing systematically in their DNSs $\left(R_{\lambda 1}=60.7-168\right)$. They observed that $C_{\varepsilon}$ depends on the external force which sustains the turbulence independently of $R_{\lambda 1}$. Kaneda et al. ${ }^{11}$ performed high resolution DNSs up to $R_{\lambda 1}=1201$. They noted that while the magnitude of $C_{\varepsilon}$ can vary with forcing for $R_{\lambda 1}<250$, it tends to a constant value $(0.4-0.5)$ at higher $R_{\lambda 1}$.

For the scalar field, some information has also been gleaned for the normalized parameter,

$$
C_{\varepsilon \theta}=\overline{\varepsilon_{\theta}} \ell / v \phi^{2}
$$

based on the expectation that, like $\bar{\varepsilon}, \overline{\varepsilon_{\theta}}$ should become constant when $R_{\lambda 1} \rightarrow \infty$, where

$$
\overline{\varepsilon_{\theta}}=\kappa \overline{\theta,{ }_{, i} \theta_{i}}
$$

is the mean scalar dissipation rate of the temperature variance $k_{\theta}\left(\equiv \overline{\theta^{2}} / 2\right)$ ( $\kappa$ is the thermal diffusivity and $\theta$ the temperature fluctuation) (e.g., Lumley, ${ }^{9} \mathrm{Xu}$ et al., ${ }^{16}$ Watanabe and Gotoh, ${ }^{17}$ and Donzis et al. ${ }^{18}$ ). By similarity to Eq. (3), the non-dimensional form

$$
C_{\varepsilon \theta}=\overline{\varepsilon_{\theta}} L_{u u} / u^{\prime} \theta^{\prime 2}
$$

follows from (6) when $\ell=L_{u u}, v=u^{\prime}$, and $\phi=\theta^{\prime}$. Whilst $C_{\varepsilon \theta}$ may approach a constant at high $R_{\lambda 1}$, there is evidence to suggest that its magnitude depends on $\operatorname{Pr}(\equiv v / \kappa)$ (the molecular Prandtl number) or Sc (the Schmidt number) (Watanabe and Gotoh ${ }^{17}$ and Donzis et al ${ }^{18}$ ) and initial conditions (Donzis et al. ${ }^{18}$ ) when $R_{\lambda 1}$ is small.

In wall turbulence, $\bar{\varepsilon}$ and $\overline{\varepsilon_{\theta}}$ may also be described independently of the viscosity away from the wall. As for other turbulent flows, the most likely normalized parameters should be Eqs. (3) and (8) although other non-normalized forms may apply in the overlap (logarithmic) region. If it is assumed that $\ell=\kappa_{u} y$ and $v=u_{\tau}\left(u_{\tau}\right.$ and $\kappa_{u}$ denote the friction velocity and the von Kármán constant, respectively), then

$$
C_{\varepsilon E Q}=\bar{\varepsilon} y / u_{\tau}^{3}=1 / \kappa_{u}
$$

(see also McKeon and Morrison ${ }^{19}$ ). Similarly, with $\ell=\kappa_{\theta} y$ and $\phi=T_{\tau}\left(T_{\tau}\right.$ and $\kappa_{\theta}$ denote the friction temperature and the von Kármán constant in the mean temperature distribution, respectively), we obtain

$$
C_{\varepsilon \theta E Q}=\overline{\varepsilon_{\theta}} y / u_{\tau} T_{\tau}^{2}=1 / \kappa_{\theta} .
$$

Equations (9) and (10) imply a relationship between the normalized parameters and the von Kármán constants. It should be noted that underpinning Eqs. (9) and (10) is the concept of local energy equilibrium (viz., $P_{k}=\bar{\varepsilon}$ and $\left.P_{\theta}=\overline{\varepsilon_{\theta}}\right)\left(P_{k}\right.$ and $P_{\theta}$ are the mean production rates for $k$ and $k_{\theta}$ ). Experimentally, the latter assumption allows one to infer $\bar{\varepsilon}$ and $\overline{\varepsilon_{\theta}}$ indirectly (and accurately) from measured mean velocity and temperature distributions. It also leads to model parameters

$$
C_{D}=C_{\mu}=u_{\tau}^{4} / k^{2}
$$

in one- and two-equation models, viz.,

$$
\bar{\varepsilon}=C_{D} \sqrt{k}^{3} / \ell
$$

and the turbulent eddy viscosity,

$$
v_{t}=C_{\mu} k^{2} / \bar{\varepsilon} .
$$

$C_{D}$ and $C_{\mu}$ are hence associated with the normalized parameters; these magnitudes are often taken as 0.09 [this can also be estimated via Eq. (11)]. $C_{D}$ and $C_{\mu}$ are also related to $C_{\lambda}$ in the eddy diffusivity model

$$
\kappa_{t}=c_{\lambda}\left(k^{2} / \bar{\varepsilon}\right) R^{p}
$$

where

$$
R=\left(k_{\theta} / \overline{\varepsilon_{\theta}}\right) /(k / \bar{\varepsilon}) .
$$

Note that $R$ denotes the time scale ratio of the temperature variance $k_{\theta} / \overline{\varepsilon_{\theta}}$ to the turbulent kinetic energy $k / \bar{\varepsilon}$; near-wall damping functions are omitted in Eqs. (13) and (14). Nagano and $\operatorname{Kim}^{20}$ used $p=1 / 2$, while Yoshizawa ${ }^{21}$ used $p=2$. Introducing the turbulent Prandtl number $\operatorname{Pr}_{t}\left(\equiv v_{t} / \kappa_{t}\right)$ leads to a relationship between $C_{\mu}$ and $C_{\lambda}$, viz., 


$$
\operatorname{Pr}_{t}=\left(C_{\mu} / C_{\lambda}\right) R^{-p} .
$$

Figure 1 suggests that energy equilibrium is indeed a better approximation for a turbulent channel flow than a turbulent boundary layer, thus implying that Eqs. (9) and (10) are better satisfied in the former flow. This also indicates that the magnitudes of $C_{\varepsilon}$ and $C_{\varepsilon \theta}$ may not vary significantly in the equilibrium region of the channel since there is little spatial flux in the $y$ direction. These normalized parameters, while essential for both physical and modeling aspects, are however yet to be examined comprehensively.

Direct numerical simulations, with good spatio-temporal resolution, have become indispensible for studying the properties of the small-scale turbulent motion. Most of the available DNS databases for wall-bounded flows have been for a turbulent channel flow, mainly due to the simple geometry of this flow. The relevant Reynolds number (or Kármán number) $h^{+}$( $\equiv u_{\tau} h / v ; u_{\tau}$ is the friction velocity and $h$ the channel halfwidth; the superscript denotes normalization by wall variables) currently extends to 2000 (Hoyas and Jiménez ${ }^{22}$ ). The
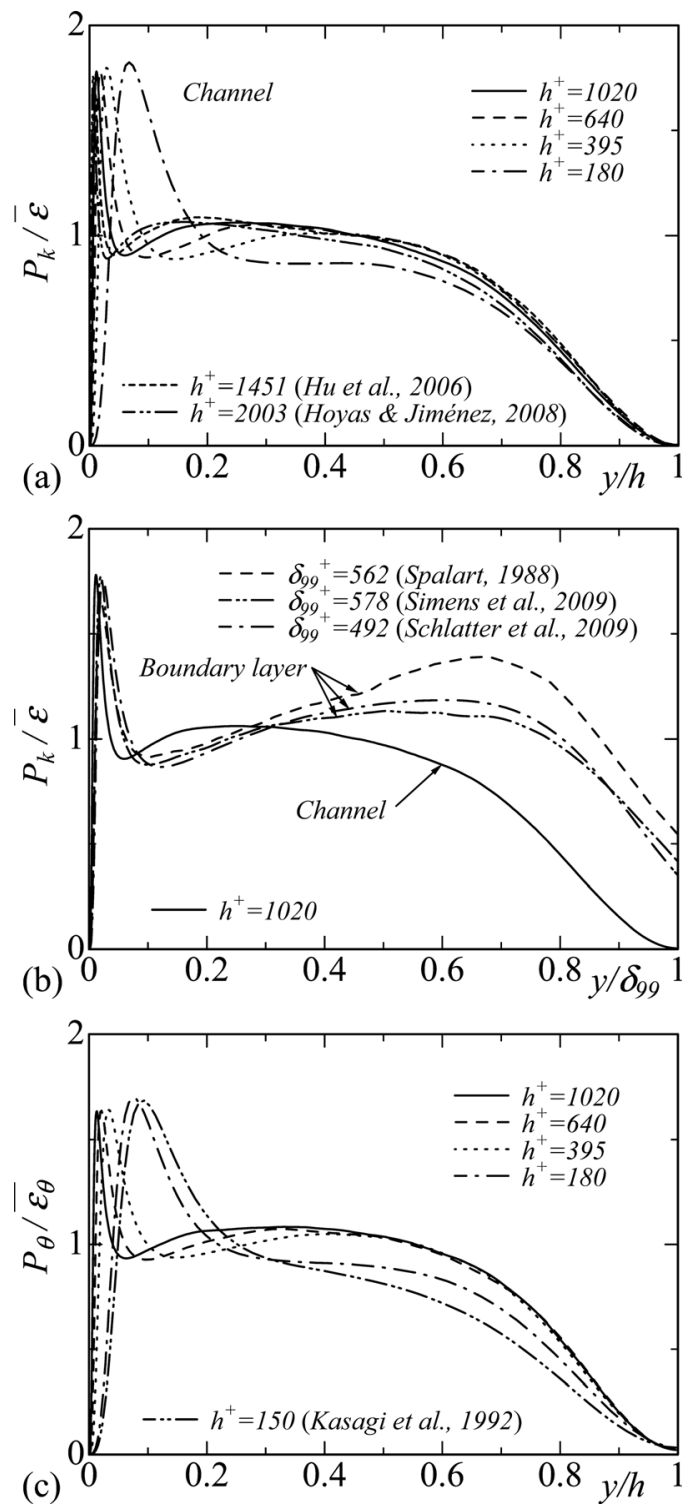

FIG. 1. Distributions of $P_{k} / \bar{\varepsilon}$ and $P_{\theta} / \overline{\varepsilon_{\theta}}$ : (a), (b) $P_{k} / \bar{\varepsilon}$; (c) $P_{\theta} / \overline{\varepsilon_{\theta}}$. channel databases have also provided a wealth of information for the small scale characteristics of turbulence (e.g., Kim and Antonia $^{23}$ and Antonia and Kim. ${ }^{24}$ ) In the outer region, when $\operatorname{Pr}$ is close to unity, both $\varepsilon$ (Blackburn et al. ${ }^{25}$ ) and $\varepsilon_{\theta}$ (Abe et $a .^{26}$ ) isotropic turbulence both statistically and instantaneously (see also the spectral and physical analogy by Antonia et $\left.a l .{ }^{27}\right)$. There is also evidence that $\bar{\varepsilon}$ scales reasonably well on $u_{\tau}^{3} / y$ [viz., Eq. (9)] and $u_{\tau}^{3} / h$ in the logarithmic and outer regions, respectively (Hoyas and Jiménez ${ }^{22}$ ).

Whilst several features of $\bar{\varepsilon}$ and $\overline{\varepsilon_{\theta}}$, including possible scaling laws, have been examined with the use of DNS data for a turbulent channel flow, the normalized parameters have yet to be tested rigorously. The main objective of this paper is to clarify the dependence on the distance from the wall and the Reynolds number of Eqs. (3) and (8) and the degree of similarity to other turbulent flows using our DNS databases (Abe et $a l .^{26,28}$ ) with $\operatorname{Pr}=0.71$. The relationships between Eqs. (3) and (9) and between Eqs. (8) and (10) will also be investigated. Particular attention is given to part of the outer region where viscous effects are small and energy equilibrium (viz., $P_{k}=\bar{\varepsilon}$ and $P_{\theta}=\overline{\varepsilon_{\theta}}$ ) represents a good approximation. It is hoped that the present investigation will shed some light on the scaling laws of $\bar{\varepsilon}$ and $\overline{\varepsilon_{\theta}}$ in the equilibrium region of a turbulent channel flow and should hence be useful for developing turbulence models [viz., Eqs. (12)(14)] given the relationship between the normalized parameters and the model parameters.

The paper is organized as follows. The present DNS databases are described briefly in Sec. II. In Sec. III, the normalized energy and scalar dissipation rates [viz., Eqs. (3) and (8)] are examined across the channel, with an emphasis on the logarithmic and outer regions and the effect of $L_{u u}$ on the magnitudes of $C_{\varepsilon}$ and $C_{\varepsilon} \theta$. In Sec. IV, we consider how the normalized parameters relate to the timescale ratio of the scalar variance to the turbulent kinetic energy $R$ [viz., Eq. (15)] and a simple relationship between the longitudinal Taylor microscale $\lambda_{1}$ and the Corrsin microscale for the temperature fluctuation $\lambda_{\theta}\left(\equiv \theta^{\prime} / \theta,_{1}^{\prime}\right)$ is proposed. In Sec. V, an attempt is made to formulate a dependence of $R_{\lambda 1}$ and $P e\left(\equiv u^{\prime} \lambda_{\theta} / \kappa\right)$ on $h^{+}$in the outer region, using values of $C_{\varepsilon}, C_{\varepsilon \theta}, u^{\prime}$, and $L_{u u}$. We also consider the applicability of the proposed form of $R_{\lambda 1}$ to the turbulent boundary layer.

\section{DNS DATABASES}

The present DNS databases have been obtained from simulations with passive scalar transport by Abe et al. ${ }^{26,28}$ The flow is a fully developed turbulent channel flow driven by a constant mean streamwise pressure gradient. The passive scalar (temperature) is introduced through uniform heating from both walls. Four values of $h^{+}(=180,395$, 640, and 1020) are used. The molecular Prandtl number $(\mathrm{Pr})$ is 0.71 .

The governing equations for the velocity and scalar fields consist of the Navier-Stokes and energy (conservation) equations, respectively. For the latter, a constant time-averaged heat-flux thermal boundary condition (Kasagi et al. ${ }^{29}$ ) is used. This condition is sometimes referred to as the isoflux 
TABLE I. Domain size, grid points, and spatial resolution.

\begin{tabular}{lcccc}
\hline \hline$h^{+}$ & 180 & 395 & 640 & 1020 \\
\hline$L_{x} \times L_{y} \times L_{z}$ & & $2.8 h \times 2 h \times 6.4 h$ & \\
$L_{x}{ }^{+} \times L_{y}{ }^{+} \times L_{z}{ }^{+}$ & $2304 \times 360 \times 1152$ & $5056 \times 790 \times 2528$ & $8192 \times 1280 \times 4096$ & $13056 \times 2040 \times 6528$ \\
$N_{x} \times N_{y} \times N_{z}$ & $768 \times 128 \times 384$ & $1536 \times 192 \times 768$ & $2048 \times 256 \times 1024$ & $2048 \times 448 \times 1536$ \\
$\Delta x^{+}, \Delta y^{+}, \Delta z^{+}$ & $3.00,0.20-5.90,3.00$ & $3.29,0.15-6.52,3.29$ & $4.00,0.15-8.02,4.00$ & $6.38,0.15-7.32,4.25$ \\
$\Delta x^{*}{ }^{*}, \Delta y_{w}{ }^{*}, \Delta z_{w}{ }^{*}$ & $1.94,0.13,1.94$ & $2.24,0.10,2.24$ & $2.77,0.11,2.77$ & $4.46,0.11,2.97$ \\
$\Delta x_{c}{ }^{*}, \Delta y_{c}{ }^{*}, \Delta z_{c}{ }^{*}$ & $0.82,1.62,0.82$ & $0.74,1.47,0.74$ & $0.82,1.64,0.82$ & $1.16,1.33,0.77$ \\
\hline \hline
\end{tabular}

condition, which is more realistic in experiments than the isothermal condition. Under the present condition, the bulk and wall mean temperatures increase linearly in the $x$ direction, while the wall temperature fluctuation is assumed to be zero. This yields a relation

$$
\frac{\partial\left\langle T^{+}\right\rangle}{\partial x^{\#}}=\frac{\partial\left\langle T_{m}^{+}\right\rangle}{\partial x^{\#}}=\frac{\partial\left\langle T_{w}^{+}\right\rangle}{\partial x^{\#}}=\frac{2}{\int_{0}^{2} \bar{U}_{1} d y^{\#}}
$$

for the present configuration $\left(T, T_{m}\right.$, and $T_{w}$ are the local, bulk, and wall temperatures, respectively; the angular bracket represents integration over $z$ and $t$ (time); the superscript \# denotes the normalization by the channel half-width $h$ [note that $t$ is normalized by both $u_{\tau}$ and $h$ ]). In the simulations of Refs. 26 and 28, an instantaneous temperature difference $\Theta$ defined by

$$
T=\frac{\partial\left\langle T_{w}\right\rangle}{\partial x} x-\Theta
$$

is applied to the energy equation, which leads to the transformed energy equation expressed as

$$
\frac{\partial \Theta^{+}}{\partial t^{\#}}+U_{j}^{+} \frac{\partial \Theta^{+}}{\partial x_{j}^{\#}}=\frac{1}{h^{+} \cdot \operatorname{Pr}} \frac{\partial^{2} \Theta^{+}}{\partial x_{j}^{\# 2}}+U_{1} \frac{2}{\int_{0}^{2} \bar{U}_{1} d y^{\#}},
$$

where $U_{i}$ is the instantaneous velocity in the $i$ th direction (see also Ref. 29). The last term of Eq. (19) comes from relation (17) and plays a role in keeping the time-averaged heat-flux constant across the channel. As in the case of the Navier-Stokes equations, the no-slip and periodic boundary conditions are used for Eq. (19) in the $y$ and other ( $x$ and $z$ ) directions, respectively.

The present thermal boundary condition differs only slightly from the internal source heating condition used by Kim and Moin. ${ }^{30}$ In the latter case, the last term of Eq. (19) is set to be 2 so that the time-averaged heat-flux is not constant across the channel, although the wall temperature fluctuation is zero. Kasagi et al. $^{29}$ noted that basic turbulence statistics and turbulence structures obtained from these two simulations should be nearly identical.

The domain size $\left(L_{x} \times L_{y} \times L_{z}\right)$, number of grid points $\left(N_{x} \times N_{y} \times N_{z}\right)$, and spatial resolution $(\Delta x, \Delta y, \Delta z)$ are given in Table I. Note that the superscript * represents normalization by either $\eta, v_{K}\left(\equiv(v \bar{\varepsilon})^{1 / 4}\right)$ (Kolmogorov velocity scale), or $\theta_{B}\left(\equiv\left(\overline{\varepsilon_{\theta}} v^{1 / 2} \bar{\varepsilon}^{-1 / 2}\right)^{1 / 2}\right)$ (Batchelor temperature scale) and the subscripts $w$ and $c$ refer to values at the wall and centerline, respectively. Since $\operatorname{Pr}$ is smaller than 1 in the present case, the Batchelor length scale, $\eta_{B}\left(\equiv \eta P r^{-1 / 2}\right)$, is slightly larger than the Kolmogorov scale. The spatial resolution of the simulations is sufficient to describe adequately the behavior of all the scales of the flow up to the largest value of $h^{+}$considered here (see also Abe et al. ${ }^{26,28}$ and Antonia et $\left.a l .{ }^{27}\right)$.

\section{NORMALIZED ENERGY AND SCALAR DISSIPATION RATES}

Figure 1 shows that there is an extended region, viz., $y^{+}=30$ to $y / h=0.7$, where the ratios $P_{k} / \bar{\varepsilon}$ and $P_{\theta} / \overline{\varepsilon_{\theta}}$ are close to 1 , at least when $h^{+}$is sufficiently large (the DNS data in the present flow by Hoyas and Jiménez ${ }^{22}$ and $\mathrm{Hu}$ et $a l .^{31}$ for $P_{k} / \bar{\varepsilon}$ and those by Kasagi et al. ${ }^{29}$ for $P_{\theta} / \overline{\varepsilon_{\theta}}$ are included for comparison). This region could therefore be interpreted as a region where energy equilibrium is validated approximately since the mean production and dissipation rates of the turbulent kinetic energy are approximately equal. Note that this region extends beyond that which is normally referred to as the log-law region. This seems to be associated with the presence of large-scale structures of $u$ and $\theta$ spanning the channel where the intense sites of $\varepsilon$ and $\varepsilon_{\theta}$ are most likely to coincide with the large-scale anisotropic $u$ and $\theta$ structures, respectively (see, for example, Abe et $a .^{26}$ and Antonia et $a l^{27}$ ). Such an equilibrium region is less evident in a turbulent boundary layer (the DNS data for $P_{k} / \bar{\varepsilon}$ of Spalart, ${ }^{32}$ Simens et al., ${ }^{33}$ and Schlatter et $a l .{ }^{34}$ are included in Fig. 1(b), where $\delta_{99}$ is used as the outer representative length scale). The difference appears to be mainly due to the importance of the advection term in the outer region of the boundary layer. This also implies that indirect estimates of $\bar{\varepsilon}$ and $\overline{\varepsilon_{\theta}}$ from $P_{k}$ and $P_{\theta}$ would be less accurate in the latter flow.

In order to assess more precisely the extent of the equilibrium region, the magnitudes of the diffusion terms in the budgets of $k$ and $k_{\theta}$ has also been examined. The transport equation of $k$, normalized by $u_{\tau}^{4} / v$, is written as

$$
\begin{aligned}
0= & \underbrace{-\overline{u_{i}^{+} u_{j}^{+}} \frac{\partial \bar{U}_{i}^{+}}{\partial x_{j}^{+}}}_{1}-\underbrace{-\frac{\partial}{\partial x_{j}^{+}}\left(\frac{1}{2} \overline{u_{i}^{+2} u_{j}^{+}}\right)}_{3} \underbrace{-\frac{\partial}{\partial x_{j}^{+}}\left(\frac{1}{2} \overline{u_{i}^{+} p^{+}}\right)}_{4} \\
& \underbrace{\frac{\partial^{2}}{\partial x_{j}^{+2}}\left(\frac{1}{2} \overline{u_{i}^{+2}}\right)}_{5}-\underbrace{\left(\frac{\partial u_{i}^{+}}{\partial x_{j}^{+}}\right)}_{3},
\end{aligned}
$$

where terms 1, 2, 3, 4, and 5 denote the production, turbulent diffusion, pressure diffusion, molecular diffusion, and homogeneous dissipation rate, respectively. Note that in Eq. (20), the homogeneous dissipation rate $\bar{\varepsilon}_{\text {hom }}$ is used instead of the 
full dissipation rate $\bar{\varepsilon}$. Also, the transport equation of $k_{\theta}$, normalized by $u_{\tau}^{2} T_{\tau}^{2} / v$, is

$$
\begin{aligned}
0= & \underbrace{-\overline{\theta^{+} u_{j}^{+}} \frac{\partial \bar{\Theta}^{+}}{\partial x_{j}^{+}}+\overline{\theta^{+} u_{1}^{+}} \frac{\partial\left\langle\bar{T}_{m}\right\rangle^{+}}{\partial x_{1}^{+}}}_{1} \underbrace{-\frac{\partial}{\partial x_{j}^{+}}\left(\frac{1}{2} \overline{\theta^{+2} u_{j}^{+}}\right)}_{2} \\
& +\underbrace{\frac{1}{\operatorname{Pr} \frac{\partial^{2}}{\partial x_{j}^{+2}}\left(\frac{1}{2} \overline{\theta^{+2}}\right)}-\underbrace{\frac{1}{\operatorname{Pr}\left(\frac{\partial \theta^{+}}{\partial x_{j}^{+}}\right)^{2}}}_{4},}_{3}
\end{aligned}
$$

where terms $1,2,3$, and 4 are the production, turbulent diffusion, molecular diffusion, and dissipation rate, respectively. Figure 2 displays that the turbulent and pressure diffusions are actually negligible only in the region $y^{+}=100$ to $y / h=0.7$. It seems therefore more appropriate to refer to this latter region as a "near-equilibrium region." With the exception of the latter region, the magnitudes of the diffusion terms are larger for Eq. (20) than for Eq. (21). In particular, the magnitude of the pressure diffusion term in Eq. (20), which is absent in Eq. (21), is appreciable for $y^{+}<100$. This suggests that the scalar field is closer to an equilibrium state than the velocity field over a wider range of the channel. It should also be noted that there is weak $h^{+}$dependence on the diffusion terms for both Eqs. (20) and (21), which indicates that the region below $y^{+}=100$ is unlikely to be in energy equilibrium, at least for $h^{+}=\mathrm{O}\left(10^{2}\right)-\left(10^{3}\right)$.

Figure 3 indicates that $C_{\varepsilon}$ [Eq. (3)] and $C_{\varepsilon \theta}$ [Eq. (8)] have nearly the same magnitude in the range $y^{+}=100$ to $y / h$ $=0.7$ (DNS (Hoyas and Jiménez ${ }^{22}$ and Hu et al. ${ }^{31}$ ) and
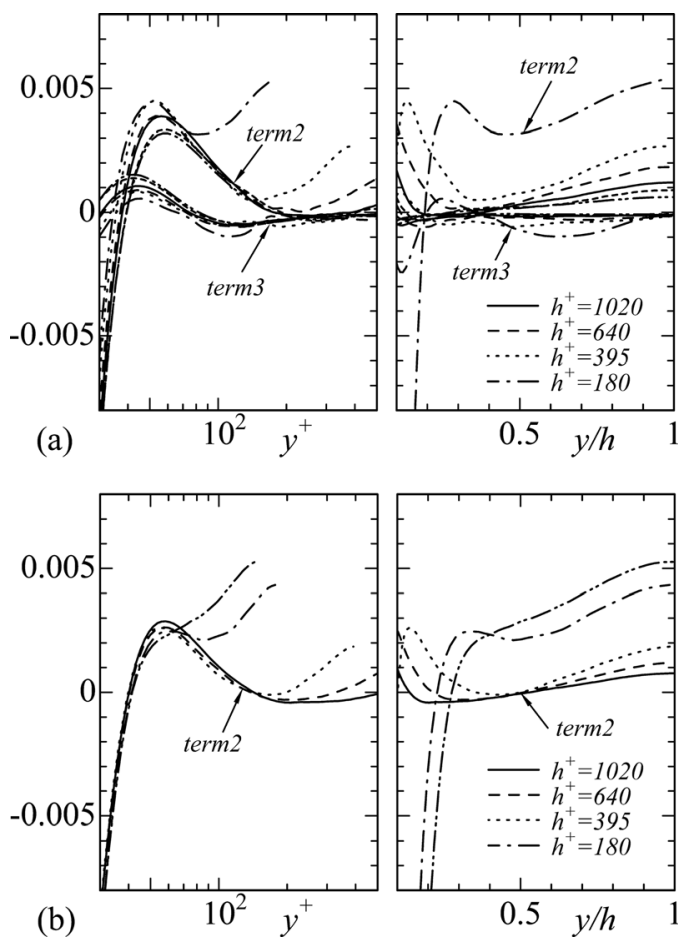

FIG. 2. Distributions of the diffusion terms in the budgets of $k$ and $k_{\theta}$ normalized on inner variables: (a) terms 2 and 3 in Eq. (20); (b) term 2 in Eq. (21). In (a),,$---- h^{+}=1451$ [Hu et al. (Ref. 31)]; - -.-..-,$h^{+}=2003$ [Hoyas and Jiménez (Ref. 22)], while in (b), - ..-..-, $h^{+}=150$ [Kasagi et al. (Ref. 29)]. experimental (Comte-Bellot ${ }^{35}$ ) data are also included) in which energy equilibrium is approximated closely. Note that when obtaining $L_{u и}$ [Eq. (4)], the integration of $R_{u и}$ has been carried out up to a separation which corresponds to the first zero crossing point (the extent of the integration is taken to $L_{x} / 2$ when $R_{u u}$ remains positive).

Outside the wall region, the magnitudes of $C_{\varepsilon}$ and $C_{\varepsilon \theta}$ decrease significantly with increasing distance from the wall up to $y / h=0.2$. Such $y$ dependence may be explained via the distributions of $\bar{\varepsilon} h / u_{\tau}^{3}, \overline{\varepsilon_{\theta}} h / u_{\tau} T_{\tau}^{2}, u^{\prime} / u_{\tau}, \theta^{\prime} / T_{\tau}$, and $L_{u u} / h$ (Figs. 4-6) since

$$
\begin{gathered}
\bar{\varepsilon} L_{u u} / u^{\prime 3}=\left(\bar{\varepsilon} h / u_{\tau}^{3}\right) \cdot\left(L_{u u} / h\right) \cdot\left(u_{\tau}^{3} / u^{\prime 3}\right), \\
\overline{\varepsilon_{\theta}} L_{u u} / u^{\prime} \theta^{\prime 2}=\left(\overline{\varepsilon_{\theta}} h / u_{\tau} T_{\tau}^{2}\right) \cdot\left(L_{u u} / h\right) \cdot\left(u_{\tau} / u^{\prime}\right) \cdot\left(T_{\tau}^{2} / \theta^{\prime 2}\right) .
\end{gathered}
$$

In that region, the magnitudes of $\bar{\varepsilon} h / u_{\tau}^{3}$ and $\overline{\varepsilon_{\theta}} h / u_{\tau} T_{\tau}^{2}$ are by one order larger than those of $u^{\prime} / u_{\tau}, \theta^{\prime} / T_{\tau}$, and $L_{u u} / h$ owing to the appreciable magnitudes of the mean velocity and temperature gradients. There is hence a similarity in shape between $C_{\varepsilon}$ and $\bar{\varepsilon} h / u_{\tau}^{3}$ [viz., Figs. 3(a) and 4(a)] and between $C_{\varepsilon \theta}$ and $\overline{\varepsilon_{\theta}} h / u_{\tau} T_{\tau}^{2}$ [viz., Figs. 3(b) and 4(b)].

In the logarithmic region (viz., $y^{+}=100$ to $y / h=0.2$ ), the values $(\sim 2)$ of $C_{\varepsilon}$ and $C_{\varepsilon \theta}$ are nearly identical with those for $C_{\varepsilon E Q}$ [Eq. (9)] and $C_{\varepsilon \theta E Q}$ [Eq. (10)] (see insets of Fig. 4) and hence with $\kappa_{u}{ }^{-1}$ and $\kappa_{\theta}{ }^{-1}$. The same values of $C_{\varepsilon}$ and $C_{\varepsilon E Q}$ are reported by Sreenivasan ${ }^{7}$ and McKeon and Morrison ${ }^{19}$ for a turbulent boundary layer and a turbulent pipe flow, respectively. It may thus be tempting to conclude that in the logarithmic region $C_{\varepsilon}$ and $C_{\varepsilon \theta}$ are identical for these three canonical flows. This equality appears to be associated with the similarity between the mean velocity and temperature distributions.
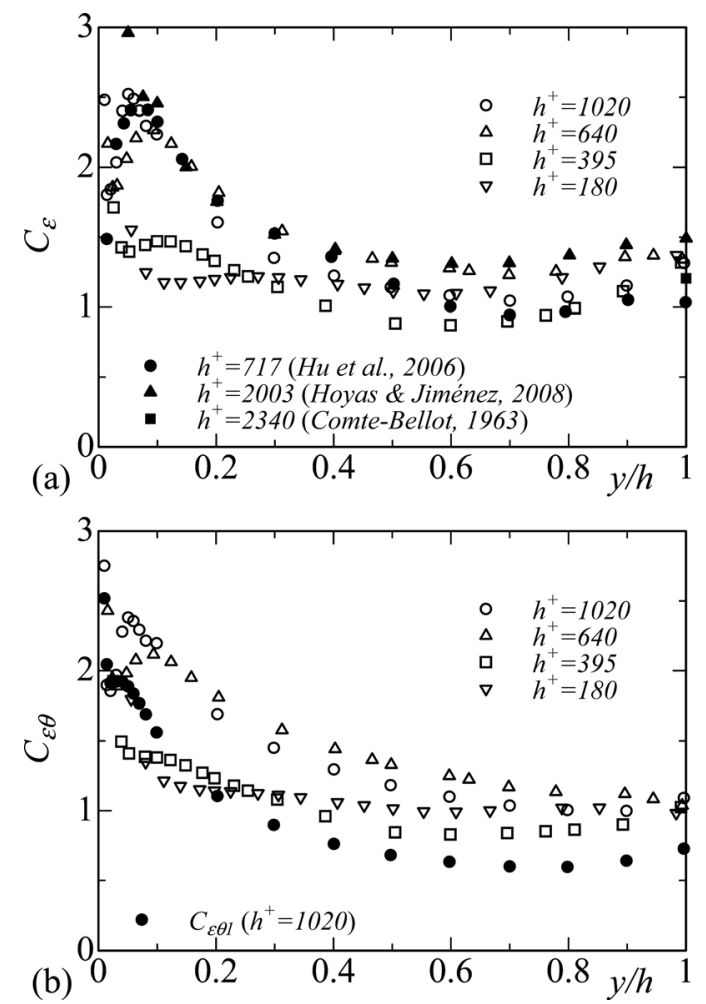

FIG. 3. Distributions of $C_{\varepsilon}$ and $C_{\varepsilon \theta}$ : (a) $C_{\varepsilon}$; (b) $C_{\varepsilon \theta}$. 

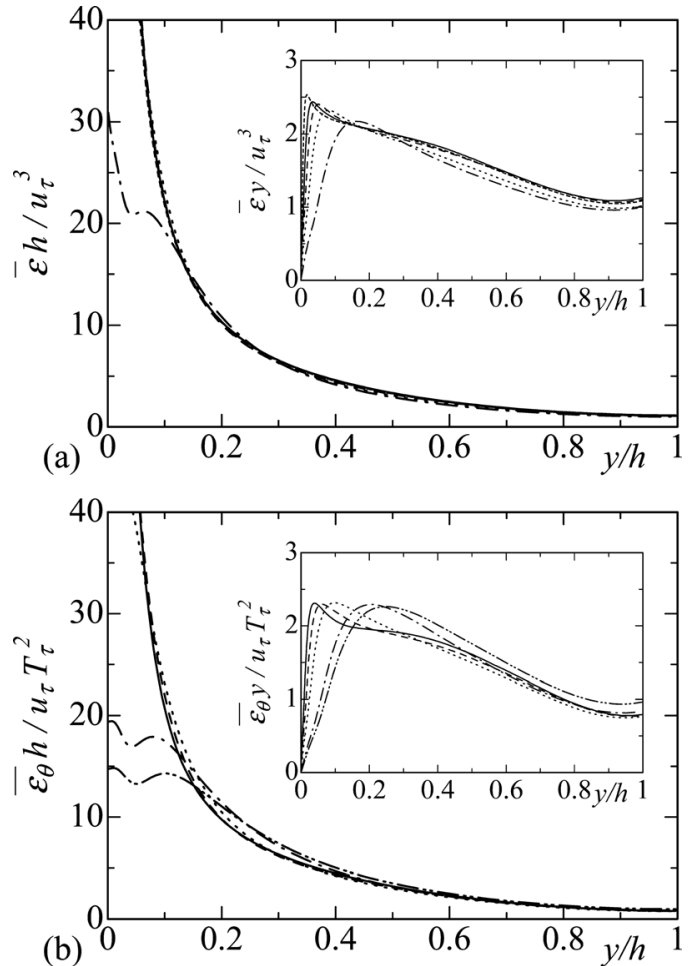

FIG. 4. Distributions of $\bar{\varepsilon} h / u_{\tau}^{3}$ (a) and $\overline{\varepsilon_{\theta}} h / u_{\tau} T_{\tau}^{2}$ (b) where the insets (a) and (b) show the distributions of $\bar{\varepsilon} y / u_{\tau}^{3}$ and $\overline{\varepsilon_{\theta}} y / u_{\tau} T_{\tau}^{2}$, respectively. $h^{+}=1020 ;----, h^{+}=640 ; \ldots \ldots \ldots, h^{+}=395 ;-.-\ldots, h^{+}=180 ;-\cdots$, $h^{+}=2003$ [Hoyas and Jiménez (Ref. 22)]; -..-..-,$h^{+}=150$ [Kasagi et al. (Ref. 29)].

For $0.3<y / h<0.7$, the variations of $C_{\varepsilon}$ and $C_{\varepsilon \theta}$ are small and their magnitudes are close to 1 . This latter value is however about twice as large as in homogeneous isotropic turbulence (Sreenivasan, ${ }^{8}$ Kaneda et al., ${ }^{11}$ Watanabe and Gotoh, ${ }^{17}$ and Donzis et $a l .{ }^{18}$ ). This is most likely associated with the existence of large-scale $u$ structures in the outer region since $L_{u u} / h$ exhibits a nearly parabolic distribution with a tendency to a plateau $(\sim 1)$ [see Fig. 6(a)], although the magnitudes of $\bar{\varepsilon} h / u_{\tau}^{3}, \overline{\varepsilon_{\theta}} h / u_{\tau} T_{\tau}^{2}, u^{\prime} / u_{\tau}$, and $\theta^{\prime} / T_{\tau}$ decrease monotonically (see Figs. 4 and 5).

The spread in the magnitudes of $C_{\varepsilon}$ and $C_{\varepsilon \theta}$ most probably reflects the difficulty in determining $L_{u и}$ accurately. Although the large-scale structures $(\sim 3 h)$ should be adequately resolved by the current DNSs, very large-scale structures with a length scale larger than $25 h$ (Hutchins and Marusic $^{36}$ and Monty and Chong ${ }^{37}$ ) are unlikely to be captured correctly. To gain further insight into this, we have integrated the two-point correlation of $u$ for two different intervals, viz.,

$$
\begin{gathered}
L_{u и 1}=\int_{0}^{3 h} R_{u и}(r) d r, \\
L_{u и 2}=\int_{3 h}^{L_{x} / 2} R_{u u}(r) d r,
\end{gathered}
$$

where $L_{u u 1}$ and $L_{u u 2}$ correspond to the contributions from the large-scale structures and the very-large-scale structures, respectively. Figure 6 suggests that while the level of $L_{u и 1}$ increases slowly with $h^{+}$in the outer region, $L_{u и 2}$ exhibits a
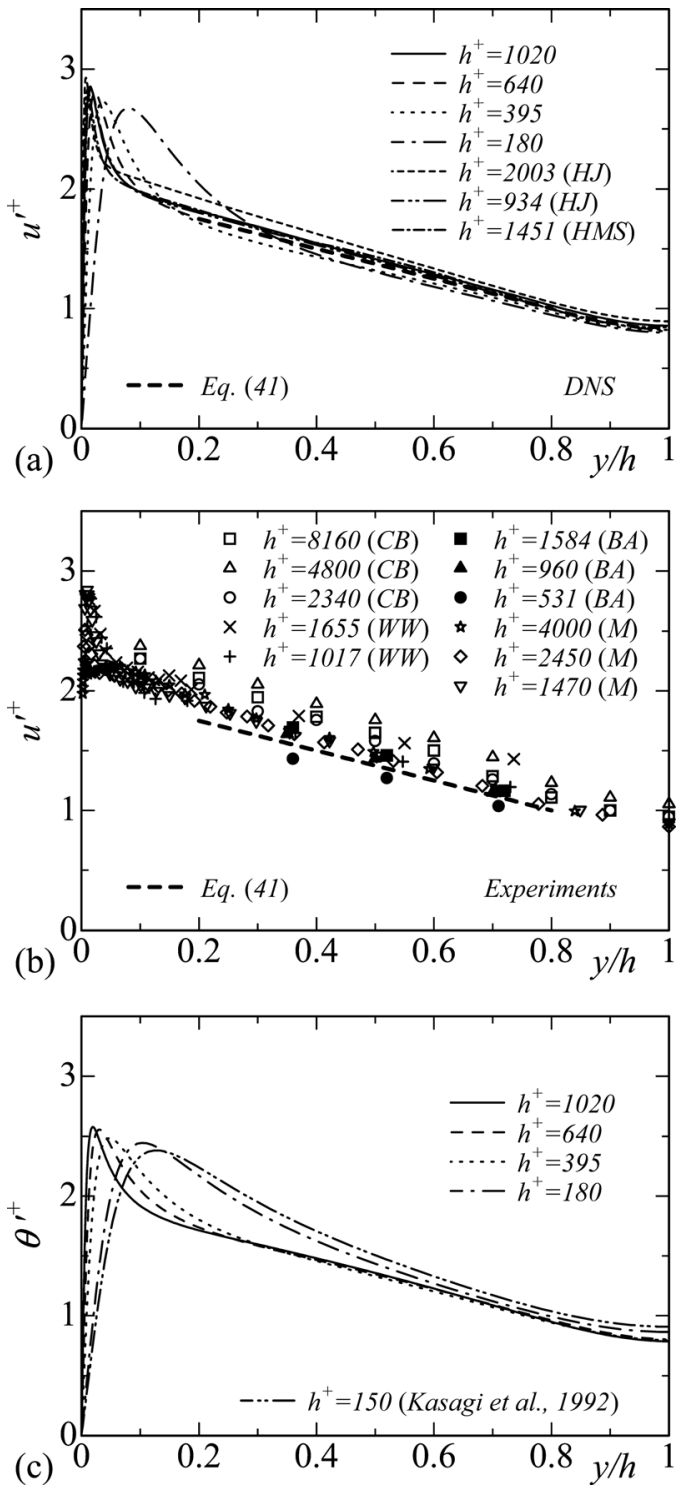

FIG. 5. Distributions of $u^{\prime+}$ and $\theta^{\prime+}$ : (a), (b) $u^{\prime+}$; (c) $\theta^{\prime+}$.

large spread. The latter is associated with the difficulty in obtaining the data at large separations accurately, presumably reflecting the insufficiency of either the computational domain size or the sampling time period.

The effects of $R_{\lambda 1}$ and the Kolmogorov-normalized mean shear $S^{*}\left(\equiv d \bar{U}^{*} / d y^{*}\right)$ may not be entirely dismissed in the context of the magnitudes for $C_{\varepsilon}$ and $C_{\varepsilon \theta}$. In the present flow, the magnitudes of $R_{\lambda 1}$ and $S^{*}$ do not depend noticeably on $y / h$ for a particular value of $h^{+}$in the near-equilibrium region. For a given value of $y / h, R_{\lambda 1}$ increases whilst $S^{*}$ decreases as $h^{+}$increases. At $y / h=0.4$, where $R_{\lambda 1}$ exhibits a local maximum [see Fig. 11(a)], the magnitude of $R_{\lambda 1}$ increases monotonically from $62\left(h^{+}=180\right)$ to 149 $\left(h^{+}=1020\right)$, whilst that of $S^{*}$ decreases systematically from $0.23\left(h^{+}=180\right)$ to $0.12\left(h^{+}=1020\right)$. From the previous observations, one may infer that, in the present flow, the effect of $R_{\lambda 1}$ and $S^{*}$ on the magnitudes of $C_{\varepsilon}$ and $C_{\varepsilon \theta}$ should be less important than that of $L_{u u}$.

In the latter context, replacing $L_{u и}$ by $L_{\theta \theta}$ in Eq. (8) yields 

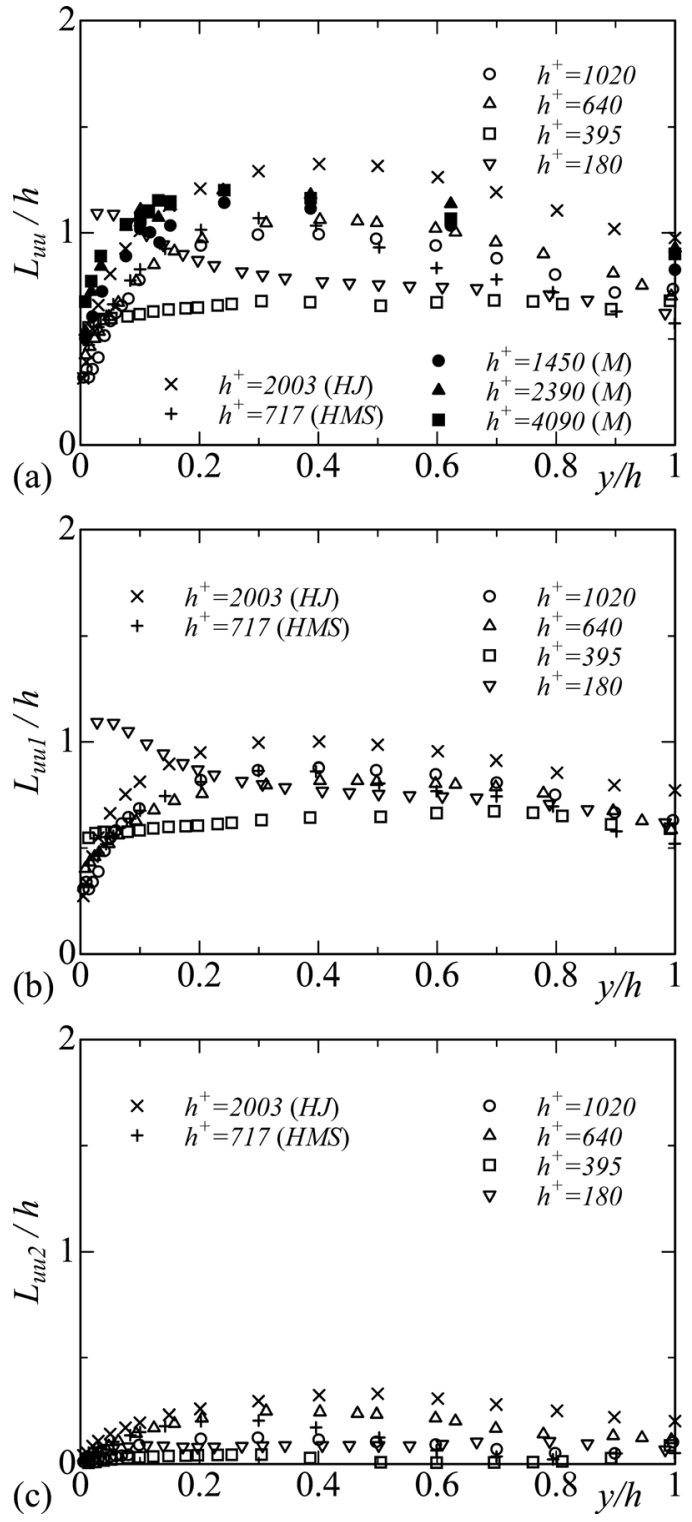

FIG. 6. Distributions of $L_{u u} / h$ : (a) $L_{u u} / h$; (b) $L_{u u 1} / h$; (c) $L_{u u 2} / h$.

$$
C_{\varepsilon \theta 1}=\overline{\varepsilon_{\theta}} L_{\theta \theta} / u^{\prime} \theta^{\prime 2}
$$

where

$$
L_{\theta \theta}=\int_{0}^{\infty} R_{\theta \theta}(r) d r
$$

This reduces the magnitude by a factor of about two [see the difference between $C_{\varepsilon \theta}$ and $C_{\varepsilon \theta 1}$ in Fig. 3(b)], reflecting the smaller extent of the large-scale $\theta$ structures relative to the large-scale $u$ structures (see Antonia et al. $^{27}$ ).

In the context of forming $C_{\varepsilon}$, there are advantages in replacing $L_{u u}$ by $L_{q q}$, viz.,

$$
L_{q q}=\int_{0}^{\infty} R_{u u}(r) d r+\int_{0}^{\infty} R_{v v}(r) d r+\int_{0}^{\infty} R_{w w}(r) d r,
$$

where $q^{2} \equiv u_{i}^{2}$. Antonia et $a .^{27}$ reported that the analogy, in both spectral and physical spaces, between $\boldsymbol{q}$ (the fluctuating velocity vector) and $\theta$ holds reasonably well throughout the

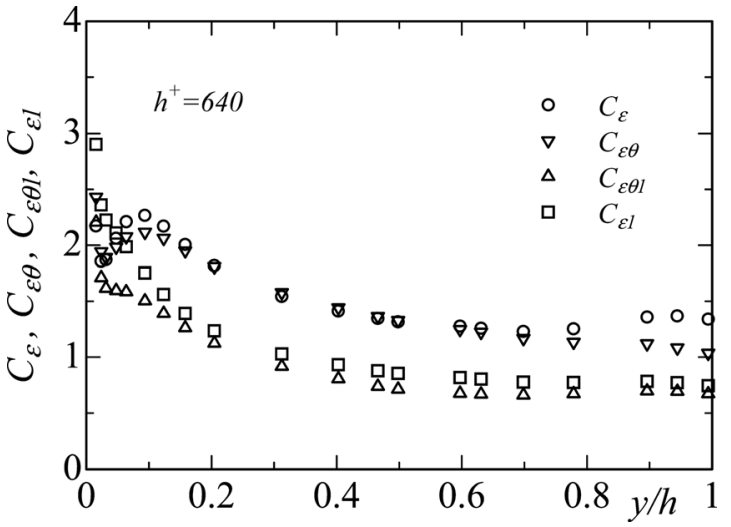

FIG. 7. Distributions of $C_{\varepsilon}, C_{\varepsilon \theta}, C_{\varepsilon}$, and $C_{\varepsilon \theta 1}$ at $h^{+}=640$.

channel when $\operatorname{Pr}$ is close to unity. This analogy suggests another possible form for the normalized mean energy dissipation rate, viz.,

$$
C_{\varepsilon 1}=\bar{\varepsilon} L_{q q} / u^{\prime} q^{\prime 2}
$$

A comparison between $C_{\varepsilon 1}$ and $C_{\varepsilon \theta 1}$ along with that between $C_{\varepsilon}$ and $C_{\varepsilon \theta}$ is made at $h^{+}=640$ and is shown in Fig. 7. Like $C_{\varepsilon}$ and $C_{\varepsilon \theta}$, the normalized parameters are in closer agreement when $L_{q q}$ and $L_{\theta \theta}$ are used in conjunction with $q^{2}$ and $\theta^{2}$ (viz., $C_{\varepsilon 1}$ and $C_{\varepsilon \theta 1}$ ) in the near-equilibrium region. The present result reflects the reasonable similarity between $q^{2}$ and $\theta^{2}$. Note that the variation of $L_{\theta \theta} / L_{q q}$ is small across the channel [Fig. 9(c)], which is another consequence of the excellent analogy between $\boldsymbol{q}$ and $\theta$.

From a turbulence modeling viewpoint, it is worth enquiring into the difference between the magnitude of $L_{u u}$ and that of the mixing length $L_{m}\left(L_{m}=\kappa_{u} y\right.$ near the wall, while $L_{m}=0.1 \mathrm{~h}$ in the outer region). In the present flow, the mixing length is more likely to be identifiable with the dissipation length scale

$$
L_{\varepsilon}=(-\overline{u v})^{3 / 2} / \bar{\varepsilon}
$$

[Fig. 8(a)] than with $L_{u и}$ [Fig. 6(a)]. Bradshaw ${ }^{38}$ also suggested that the magnitude of $L_{m}$ is nearly equal to that of $L_{\varepsilon}$ across most of the boundary layer. After substituting $L_{\varepsilon}$ and $\sqrt{k}$ for $\ell$ and $v$, respectively, in Eq. (2), we obtain another normalized parameter, viz.,

$$
C_{\varepsilon 2}=\bar{\varepsilon} L_{\varepsilon} / \sqrt{k}^{3},
$$

this relation being identical with Eq. (12). Figure 8(b) shows that $C_{\varepsilon 2} \sim 0.1$ over the equilibrium region. This value is not far from $C_{D}=C_{\mu}=0.09$ in the model calculations, which leads to reasonable model predictions in the present flow.

\section{RELATIONSHIP BETWEEN THE NORMALIZED PARAMETERS AND TIME SCALE RATIO}

The approximate equality of $C_{\varepsilon}$ and $C_{\varepsilon \theta}$ in the region $y^{+}=100$ to $y / h=0.7$ has important implications for the time scale ratio $R$. By substituting Eqs. (3) and (8) into Eq. (15), we obtain 

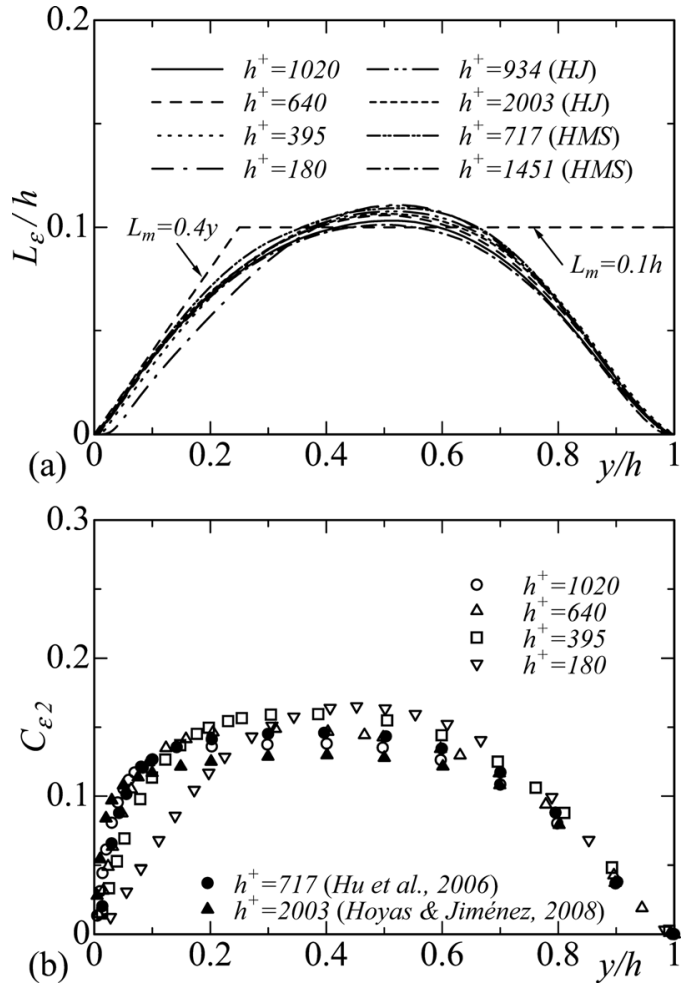

FIG. 8. Distributions of $L_{\varepsilon} / h$ and $C_{\varepsilon 2}$ : (a) $L_{\varepsilon} / h$; (b) $C_{\varepsilon 2}$.

$$
R=\frac{\overline{u u}}{\overline{q q}} \frac{C_{\varepsilon}}{C_{\varepsilon \theta}} .
$$

Also, the near equality of $C_{\varepsilon 1}$ [Eq. (29)] and $C_{\varepsilon \theta 1}$ [Eq. (26)] in this region leads to an alternative form

$$
R=\frac{L_{\theta \theta}}{L_{q q}} \frac{C_{\varepsilon 1}}{C_{\varepsilon \theta 1}} .
$$

Since $C_{\varepsilon} \simeq C_{\varepsilon \theta}$ and $C_{\varepsilon 1} \simeq C_{\varepsilon \theta 1}$ in this range, $R$ may be approximated in two different ways

$$
R=\overline{u u} / \overline{q q}
$$

and

$$
R=L_{\theta \theta} / L_{q q}
$$

Equations (34) and (35) are tested against the DNS data in Fig. 9, which indicates that these equations are reasonable approximations to $R$ over the region $y^{+}=100$ to $y / h=0.7$ (one exception is $h^{+}=180$ due to the low Reynolds-number effects) for $\operatorname{Pr}=0.71$. The magnitude of $R$, however, increases slowly with increasing $P r$ in the outer region (see Kasagi and Ohtsubo, ${ }^{39}$ Kawamura et al., ${ }^{40,41}$ Schwertfirm and Manhart, ${ }^{42}$ and Kozuka et $\left.a l .{ }^{43}\right)$; for $h^{+}=395, R$ varies from $0.1(\operatorname{Pr}=0.025)$ to $1(\operatorname{Pr}=10)$. This needs to be incorporated into Eq. (34) when formulating $R$. The latter formula may allow us to exclude $R$ from Eq. (14). This may be desirable for developing the eddy diffusivity model since the inclusion of the scalar time scale in Eq. (14) violates the linearity principle of the scalar transport $\left(\mathrm{Pope}^{44}\right)$.
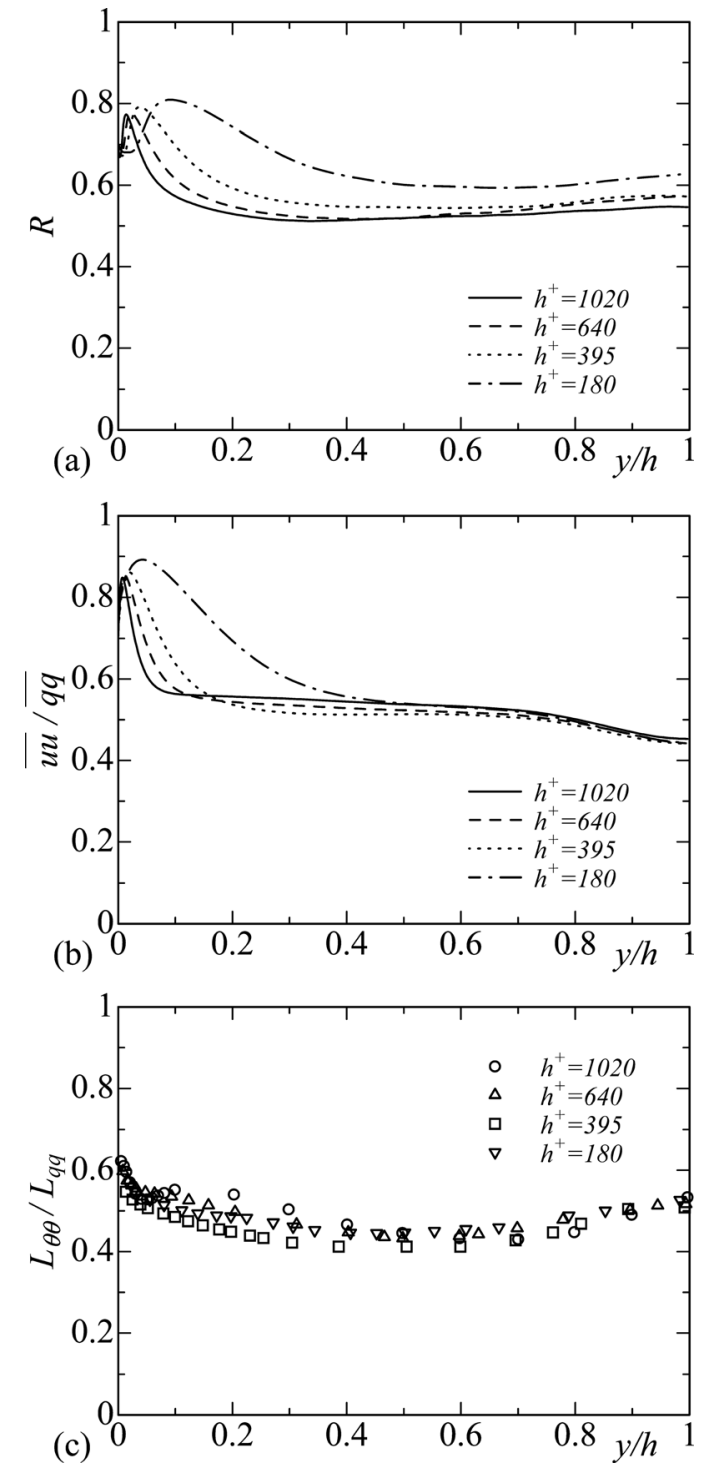

FIG. 9. Distributions of $R, \overline{u u} / \overline{q q}$, and $L_{\theta \theta} / L_{q q}$ : (a) $R$; (b) $\overline{u u} / \overline{q q}$; (c) $L_{\theta \theta} / L_{q q}$.

Equation (34) also suggests the existence of a possible relationship between $\lambda_{1}$ and $\lambda_{\theta}$. Assuming isotropic forms for $\bar{\varepsilon}$ and $\overline{\varepsilon_{\theta}}, R$ may be written as

$$
R=5 \operatorname{Pr} \frac{\overline{u u}}{\overline{q q}}\left(\frac{\lambda_{\theta}}{\lambda_{1}}\right)^{2} .
$$

Substituting Eq. (34) into Eq. (36) leads to

$$
\lambda_{\theta}=\lambda_{1} / \sqrt{5 \operatorname{Pr}} \text {. }
$$

This relation may be useful for estimating $\lambda_{\theta}$, although the Prandtl-number effect on $R$ is expected to affect the prediction of Eq. (37) to some extent. This is tested in Fig. 10 where the DNS channel flow data of Kozuka et al. ${ }^{43}\left(h^{+}=\right.$ 395 and $P r=2$ and 10) and Abe et $a l^{28}\left(h^{+}=395\right.$ and $\operatorname{Pr}=0.025)$ are included to clarify the Prandtl-number dependence. The isotropic form $\lambda_{\theta i s o}\left(\equiv \theta^{\prime} / \sqrt{\overline{\varepsilon_{\theta}} / 3 \kappa}\right)$ is also used; the isotropic relation is reasonable in the outer region, consistent with the results of Antonia et al. ${ }^{27}$ for $\overline{\varepsilon_{i s o}}$ and $\overline{\varepsilon_{\theta i s o}}$, except for $h^{+}=180$ due to the low Reynolds-number effects [see Fig. 10(a)]. In the near-equilibrium region, 

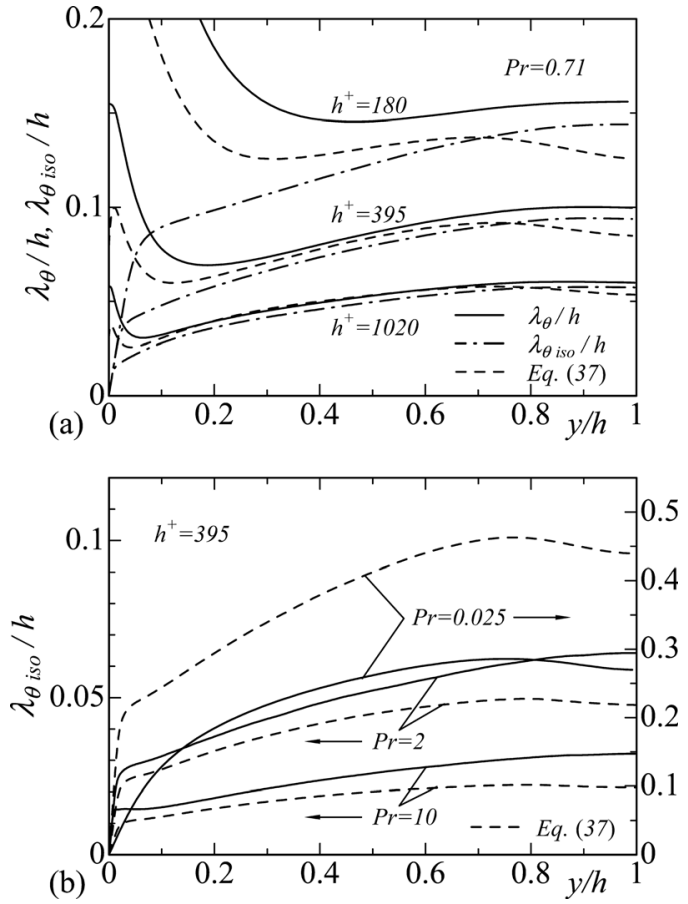

FIG. 10. Distributions of $\lambda_{\theta} / h$ and $\lambda_{\theta i s o} / h$ : (a) $\lambda_{\theta} / h$ and $\lambda_{\theta i s o} / h$ for $\operatorname{Pr}=0.71$; (b) $\lambda_{\theta i s o} / h$ for $h^{+}=395$.

Eq. (37) is not applicable for $P r=0.025$ (viz., mercury) but is likely to be valid at least for $\operatorname{Pr}=0.71-10$ (viz., air and water), the latter $P r$ range being larger than that for Eq. (34). This finding is of importance for formulating $P e$, as discussed in Sec. V.

\section{FORMULATIONS FOR $R_{\lambda 1}$ AND Pe}

Empirical expressions for the dependence on $h^{+}$of $R_{\lambda 1}$ and $P e$ in a part of the outer region (viz., $0.3<y / h<0.7$ ) can be established with the use of the approximate equality between $C_{\varepsilon}$ and $C_{\varepsilon \theta}$ in this range. With the use of the approximation $C_{\varepsilon}=C_{\varepsilon \theta}=1.2$ and $L_{u u} / h=1.2$ and the assumption of local isotropy, $R_{\lambda 1}$ and $P e$ may be expressed as

$$
R_{\lambda 1}=\frac{u^{\prime} \lambda_{1}}{v}=\sqrt{u^{\prime+}} \cdot \sqrt{\frac{15}{C_{\varepsilon}}} \cdot \sqrt{\frac{L_{u u}}{h}} \cdot \sqrt{h^{+}} \approx 3.9 \sqrt{u^{\prime+} h^{+}}
$$

and

$$
P e=\frac{u^{\prime} \lambda_{\theta}}{\kappa}=\sqrt{u^{\prime+}} \cdot \sqrt{\frac{3}{C_{\varepsilon \theta}}} \cdot \sqrt{\frac{L_{u u}}{h}} \cdot \sqrt{h^{+} P r} \approx 1.7 \sqrt{u^{\prime+} h^{+} P r},
$$

where $u^{\prime+}$ depends on $y$. Note that Eqs. (38) and (39) yield

$$
P e=\sqrt{\operatorname{Pr} / 5} R_{\lambda 1},
$$

which is analogous to the previously obtained relationship between $\lambda_{1}$ and $\lambda_{\theta}$, viz., Eq. (37).

Our DNS databases $\left(180 \leq h^{+} \leq 1020\right)$ suggest that $u^{\prime+}$ can be approximated by

$$
u^{\prime+}=-1.25(y / h)+2
$$
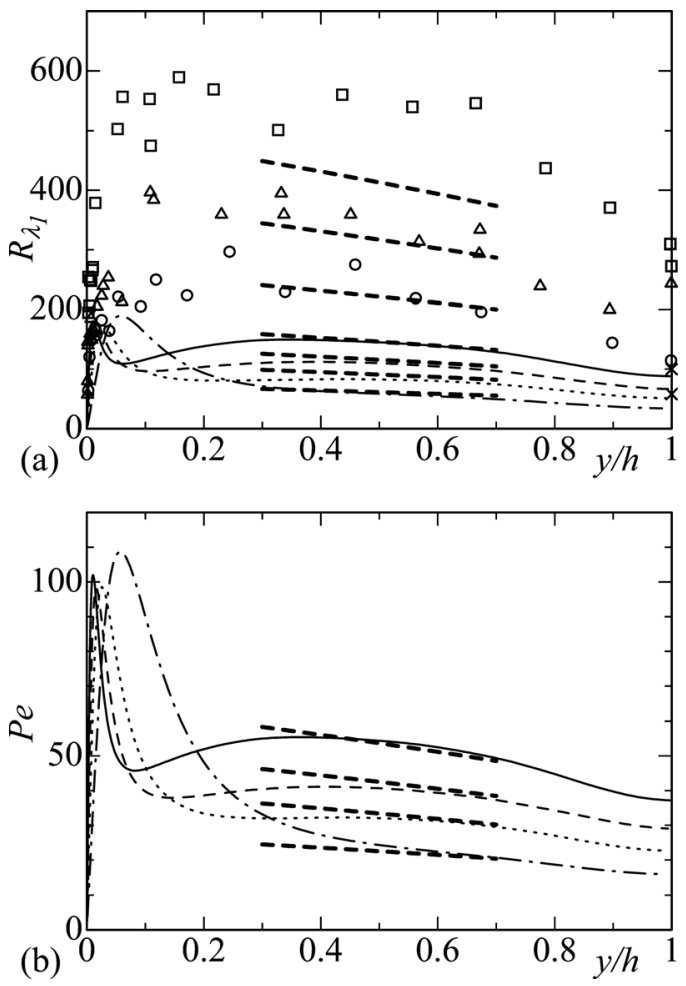

FIG. 11. Formulations for $R_{\lambda 1}$ [Eq. (38)] and $P e$ [Eq. (39)] in the region $0.3<y / h<0.7$ : (a) $R_{\lambda 1}$; (b) $P e:-, h^{+}=1020 ;---, h^{+}=640$; …......, $h^{+}=395 ;-.--, h^{+}=180 ;-\cdots-$, predictions. Symbols denote the experimental data of Comte-Bellot (Ref. 35): $\bigcirc, h^{+}=2340 ; \triangle$, $h^{+}=4800 ; \square, h^{+}=8160$.

[see Fig. 5(a)]. While a more rigorous form may be obtained by including Reynolds-number effects, Eq. (41) is reasonable at least for $h^{+}=\mathrm{O}\left(10^{2}\right) \sim\left(10^{3}\right)$. With regard to the distributions of $u^{\prime+}$, a discernible difference may be noticed between the DNS [Hoyas and Jiménez $\left.{ }^{22}(\mathrm{HJ})\right]$ and experimental [Comte-Bellot $\left.{ }^{35}(\mathrm{CB})\right]$ data at a comparable $h^{+}$. This discrepancy may be due to $u_{\tau}$ being underestimated in CB's measurements since her distributions of $\bar{U}^{+}$(not shown here) and $u^{\prime+}$ [Fig. 5(a)] for the lowest $h^{+}$are about $8 \%$ larger than those of HJ in the outer region. For the highest $h^{+}$of the CB data, the effect of compressibility may not be dismissed. It should also be noted that whilst the magnitude of $u^{\prime+}$ seems to increase slowly with $h^{+}$in the outer region, the measurements do not always indicate consistent trends for $h^{+}>1000$; CB and WW (Wei and Willmarth ${ }^{45}$ ) report larger magnitudes than BA (Balakumar and $\operatorname{Adrian}^{46}$ ) and $\mathrm{M}$ $\left(\right.$ Monty ${ }^{47}$ ), while the distributions of BA, M, and HMS (Hu et $\left.a l .{ }^{31}\right)$ are close to each other at a comparable $h^{+}$.

Equations (38) and (39) are tested in the region $0.3<y / h$ $<0.7$ in Fig. 11. For both $R_{\lambda 1}$ and $P e$, the agreement between these predictions and the DNS data is satisfactory. The poor agreement with the CB data particularly for her largest $h^{+}$ seems to be due to the limited spatial resolution of the hot wire. This attenuates small-scale intensities significantly (Hutchins et $a l .{ }^{48}$ ) and would lead to an underestimation of $u{ }_{1}^{\prime}$ (viz., $\bar{\varepsilon}$ ) and an artificial increase in $\lambda_{1}$ (viz., $R_{\lambda 1}$ ).

We focus here on the behaviors of $R_{\lambda 1}$ and $P e$ at $y / h$ $=0.4$ where $R_{\lambda 1}$ and $P e$ exhibit local maxima (see Fig. 11). At this location, Eqs. (38) and (39) can be rewritten as 

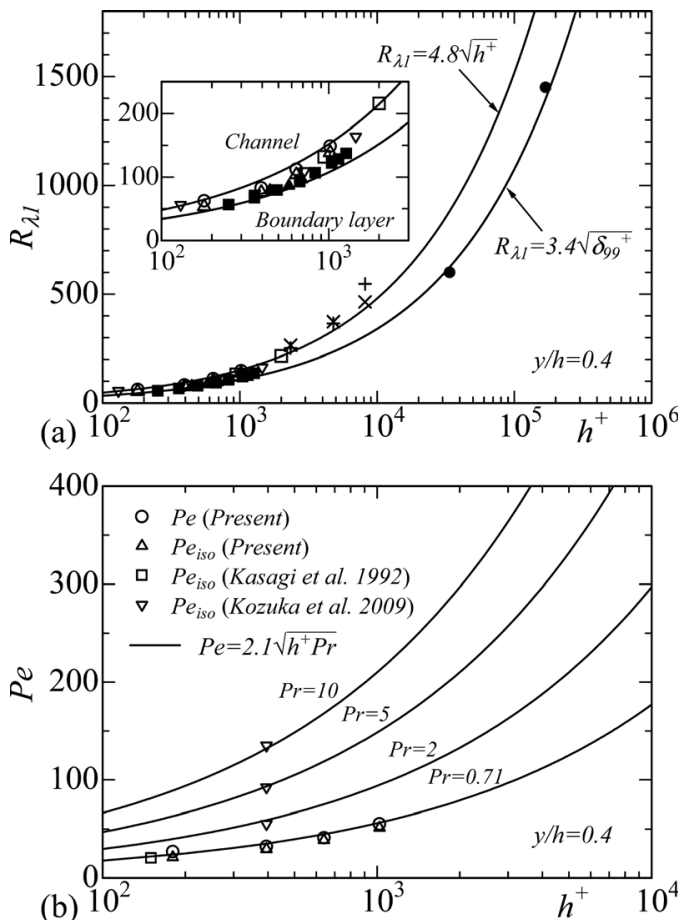

FIG. 12. Formulations for $R_{\lambda 1}$ [Eqs. (42) and (44)] and $P e$ [Eq. (43)] at $y / h=0.4$ : (a) $R_{\lambda 1}$; (b) $P e$. In (a), $\bigcirc, R_{\lambda 1}$ (Present); $\triangle, R_{\lambda 1 \text { iso }}$ (Present); $\square$, $R_{\lambda 1 \text { iso }}$ [Hoyas and Jiménez (Ref. 22)]; $\nabla, R_{\lambda 1 \text { iso }}$ [Hu et al. (Ref. 31)];,$+ R_{\lambda 1}$ [Comte-Bellot (Ref. 35)]; $\times, R_{\lambda 1}$ [Comte-Bellot (Ref. 35)] for which the correction was made; $\bullet, R_{\lambda 1}$ [Saddoughi and Veeravalli (Ref. 49)]; $\boldsymbol{\Delta}, R_{\lambda 1 \text { iso }}$ [Simens et al. (Ref. 33)]; $\mathbf{\square}, R_{\lambda 1 \text { iso }}$ [Schlatter et al. (Ref. 34)].

$$
R_{\lambda 1}=4.8 \sqrt{h^{+}}
$$

and

$$
P e=2.1 \sqrt{h^{+} P r} \text {. }
$$

The predictions of Eqs. (42) and (43) are shown in Fig. 12; the data for $R_{\lambda 1 i s o}\left(\equiv u^{\prime} \lambda_{1 i s o} / v\right)$ and $P e_{i s o}\left(\equiv u^{\prime} \lambda_{\theta i s o} / \kappa\right)$ are also included in Fig. 12. Note that $\lambda_{1 i s o} \equiv u^{\prime} / \sqrt{\bar{\varepsilon} / 15 v}$. The predicted values are in close agreement with the DNS data; there is only a small difference between $R_{\lambda 1}$ and $R_{\lambda 1 \text { iso }}$ and also between $P e$ and $P e_{i s o}$. The magnitudes of $R_{\lambda 1}$ originally given by $\mathrm{CB}$ are slightly larger than the predictions of Eq. (42) especially for her highest $h^{+}$. The CB data, however, become closer to the present predictions when a correction is applied for $\lambda_{1}$ based on the assumption $P_{k}=\bar{\varepsilon}$. For $P e$, the Prandtl-number effect seems to be reproduced reasonably well for $\operatorname{Pr}=0.71-10$, consistently with the results for $\lambda_{\theta}$ [see also Fig. 10(b)].

It seems appropriate to enquire if Eqs. (42) and (43) apply to other wall-bounded flows. The boundary layer measurements of Saddoughi and Veeravalli ${ }^{49}$ at $\delta_{99}{ }^{+}=3.4 \times 10^{4} \quad\left(y / \delta_{99} \approx 0.5\right)$ and $1.7 \times 10^{5} \quad\left(y / \delta_{99} \approx 0.4\right)$ indicate that Eq. (42) overestimates $R_{\lambda 1}$ by about $30 \%$; the latter values seem to be approximated by

$$
R_{\lambda 1}=3.4 \sqrt{\delta_{99}^{+}}
$$

[see Fig. 12(a)]. The data for $R_{\lambda 1 \text { iso }}$ by Simens et al. ${ }^{33}$ $\left(\delta_{99}{ }^{+}=445-690\right)$ and Schlatter et al. ${ }^{34}\left(\delta_{99}{ }^{+}=252-1271\right)$
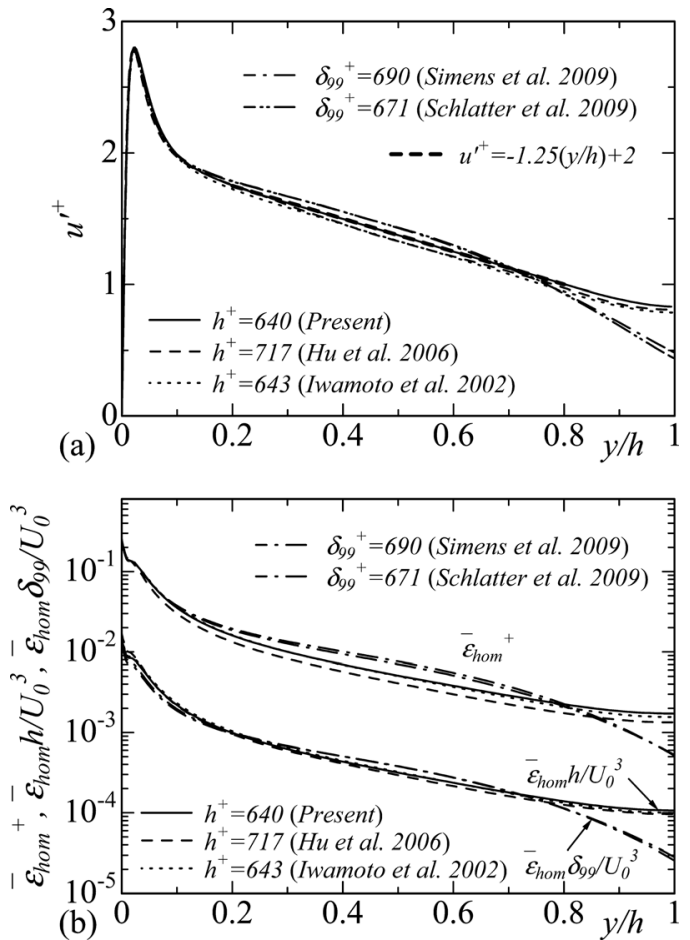

FIG. 13. Comparisons for $u^{\prime}$ and $\bar{\varepsilon}_{\text {hom }}$ between the channel flow and the boundary layer: (a) $u^{\prime+}$; (b) $\bar{\varepsilon}_{\text {hom }}$ normalized by either inner $\left(u_{\tau}{ }^{4} / v\right)$ or outer $\left[U_{0}^{3} / h\right.$ or $U_{0}^{3} / \delta_{99}\left(U_{0}\right.$ denotes either the mean centreline velocity or freestream velocity)] variables.

also agree reasonably well with Eq. (44). This appears to be due to $\bar{\varepsilon}^{+}$being about $30 \%$ larger in a boundary layer than in a channel over the equilibrium region, although the magnitude of $u^{\prime+}$ is nearly the same in the two flows. A comparison of DNS data for $u^{\prime}$ and $\bar{\varepsilon}_{\text {hom }}\left(\equiv v \overline{u_{i, j}^{2}}\right)$ (the homogeneous energy dissipation rate) between the channel flow (Abe et al., ${ }^{26} \mathrm{Hu}$ et al., ${ }^{31}$ and Iwamoto et al. ${ }^{50}$ ) and the boundary layer (Simens et al. ${ }^{33}$ and Schlatter et al. ${ }^{34}$ ) at comparable $h^{+}$and $\delta_{99}{ }^{+}$seems to confirm this (see Fig. 13 and also the comparison of $u^{\prime+}$ by Jiménez and Hoyas ${ }^{51}$ ). Note that the fall-off in the $u^{\prime}$ and $\bar{\varepsilon}_{\text {hom }}$ distributions for the turbulent boundary layer at $y / \delta_{99}>0.7$ is caused by the intermittency associated with the turbulent/non-turbulent interface. The implication is that for a given value of the Kármán number $\left(h^{+}\right.$or $\left.\delta_{99}{ }^{+}\right)$, the maximum value of $R_{\lambda 1}$ is larger for the channel flow than the boundary layer [see Fig. 12(a)]. This also suggests that the former flow may be a better candidate for examining the approach towards local isotropy than the latter.

\section{CONCLUSIONS}

DNS databases in a turbulent channel flow with passive scalar transport $\left(h^{+}=180,395,640\right.$, and 1020, $\left.\operatorname{Pr}=0.71\right)$ are used to examine the normalized mean energy and scalar dissipation rates $C_{\varepsilon}$ and $C_{\varepsilon \theta}$ [viz., Eqs. (3) and (8)]. Particular attention is given to the outer region. The main conclusions are as follows.

An interesting feature of a fully developed turbulent channel flow is the existence of a region, which starts in the inner region and extends well into the outer region of the 
channel. In this region, the ratios $P_{k} / \bar{\varepsilon}$ and $P_{\theta} / \overline{\varepsilon_{\theta}}$ are close to 1. The region $y^{+}=100$ to $y / h=0.7$, where the equality $P_{k} / \bar{\varepsilon}=P_{\theta} / \overline{\varepsilon_{\theta}}=1$ is satisfied closely and the magnitudes of the diffusion terms are negligible, can be appropriately described as a near-equilibrium region, at least for $h^{+}=\mathrm{O}\left(10^{2}\right) \sim\left(10^{3}\right)$. In this region, $\bar{\varepsilon}$ and $\overline{\varepsilon_{\theta}}$, normalized by $u^{\prime 3} / L_{u u}$ and $u^{\prime} \theta^{\prime 2} / L_{u u}$, viz., $C_{\varepsilon}$ and $C_{\varepsilon \theta}$, are approximately equal. Their magnitudes are about 2 and 1 in the logarithmic and outer regions, respectively, when the Kármán number $h^{+}$is sufficiently large. The former magnitude, which is identical with that of $\bar{\varepsilon} y / u_{\tau}^{3}$ or $\overline{\varepsilon_{\theta}} y / u_{\tau} T_{\tau}^{2}$ and hence with $\kappa_{u}{ }^{-1}$ or $\kappa_{\theta}{ }^{-1}$, tends to be approximately the same for the channel, pipe, and boundary layer. This reflects the similarity between the mean velocity and temperature distributions among these three canonical flows. When the Kármán number is large, these distributions should be relevant to the log-law (see, for example, Nagib and Chauhan ${ }^{52}$ and Marusic et al. ${ }^{53}$ ). The latter magnitude is, on the other hand, about twice as large as in homogeneous isotropic turbulence due mainly to the existence of large-scale $u$ structures in the outer region of the channel. In the present flow, the effect of $R_{\lambda 1}$ and $S^{*}$ on the magnitudes of $C_{\varepsilon}$ and $C_{\varepsilon \theta}$ is less important than that of $L_{u u}$. Replacing $L_{u u}$ by $L_{\theta \theta}$ in $C_{\varepsilon \theta}$ (viz., $C_{\varepsilon \theta 1}$ ) reduces the magnitude by a factor of about two, reflecting the smaller extent of the large-scale $\theta$ structures relative to the large-scale $u$ structures. It should also be noted that the similarity between $C_{\varepsilon 1}$ [Eq. (29)] and $C_{\varepsilon \theta 1}$ [Eq. (26)] in Fig. 7 is another consequence of the spectral analogy between $\boldsymbol{q}$ and $\theta$.

The normalized parameters are also of importance from a turbulence model standpoint. In this context, $C_{\varepsilon 2}$ [Eq. (31)], which is identical with $C_{D}$ and $C_{\mu}$, is $\sim 0.1$ over the equilibrium region. This mainly reflects the adequacy of the current turbulence models for the present flow. There is also a near equality between either $C_{\varepsilon}$ and $C_{\varepsilon \theta}$ or between $C_{\varepsilon 1}$ and $C_{\varepsilon \theta 1}$ in the near-equilibrium range, which has an important implication for the time scale ratio $R$, viz., $R$ can be approximated with Eq. (34), without any information being required about the scalar field, when the analogy between velocity and scalar fields is reasonable $(\operatorname{Pr} \approx 1)$. The magnitude of $R$, however, increases slowly with increasing $\operatorname{Pr}$ in the outer region, and this needs to be taken into account when formulating $R$. Whilst the current $k_{\theta}-\varepsilon_{\theta}$ model (e.g., Nagano and $\mathrm{Kim}^{20}$ and Yoshizawa ${ }^{21}$ ), which used $R$ in Eq. (14), gives reasonable predictions, a formula of $R$ may be useful for developing the eddy diffusivity model since it would allow the scalar time scale to be excluded from Eq. (14) and the resulting $\kappa_{t}$ model would then satisfy the linearity principle of the scalar transport $\left(\mathrm{Pope}^{44}\right.$ ).

It should be noted that the equality between $C_{\varepsilon}$ and $C_{\varepsilon \theta}$ also leads to a simple relationship between $\lambda_{1}$ and $\lambda_{\theta}$, viz., Eq. (37). Note that Eq. (37) is likely to be applicable at least for $P r=0.71-10$ (viz., air and water), the latter $\operatorname{Pr}$ range being larger than that for Eq. (34). This equality can in turn be used to establish analogous expressions for the dependence on $h^{+}$of $R_{\lambda 1}$ and $P e$ in the outer region. The proposed formulations for $R_{\lambda 1}$ and $P e$, as given by Eqs. (38) and (39) and also Eqs. (42) and (43), should be quite accurate for the channel flow (see Figs. 11 and 12). As shown in Fig. 12(a), similar formulations with slightly different numerical constants should however be used in the boundary layer since the magnitude of $R_{\lambda 1}$ is about $30 \%$ larger in the channel flow than in the boundary layer for the same Kármán number $\left(h^{+}\right.$or $\left.\delta_{99}{ }^{+}\right)$due to $\bar{\varepsilon}^{+}$being larger in the latter flow by nearly the same amount. The energy dissipation rate in a pipe flow is presumably closer to that in a channel flow than in a boundary layer since in the outer region there seems to be some structural similarity between the channel and pipe but not between the channel (or pipe) and the boundary layer (see Monty et al. ${ }^{54}$ ). The formulations established for a channel flow should hence be applicable to a pipe flow.

\section{ACKNOWLEDGMENTS}

Computations performed on JAXA (Japan Aerospace Exploration Agency) Supercomputer System are gratefully acknowledged. H.A. was partially supported by the Ministry of Education, Culture, Sports, Science and Technology of Japan, Grant-in-Aid for Young Scientists (B), Grant No. 20760125, 2009 and also the NORDITA-KTH research program on the turbulent boundary layer in 2010. R.A.A. acknowledges the support of the Australian Research Council.

${ }^{1}$ G. I. Taylor, "Statistical theory of turbulence," Proc. R. Soc. London, Ser. A 151, 421 (1935).

${ }^{2}$ A. N. Kolmogorov, "Dissipation of energy in the locally isotropic turbulence," Dokl. Akad. Nauk SSSR 32, 19 (1941) [reprinted in Proc. R. Soc. London, Ser. A 434, 15 (1991)].

${ }^{3}$ K. R. Sreenivasan and R. A. Antonia, "The phenomenology of small-scale turbulence," Annu. Rev. Fluid Mech. 29, 435 (1997).

${ }^{4}$ B. R. Pearson, T. A. Yousef, N. E. L. Haugen, A. Brandenburg, and P.-Å. Krogstad, "The 'zeroth law' of turbulence in steady isotropic turbulence," in 15th Australasian Fluid Mechanics Conference, edited by M. Behnia, W. Lin, and G. D. McBain (The University of Sydney, Australia, 2004), paper N. AFMC00040.

${ }^{5} \mathrm{G}$. K. Batchelor, The Theory of Homogeneous Turbulence (Cambridge University Press, Cambridge, England, 1953).

${ }^{6} \mathrm{~K}$. R. Sreenivasan, "On the scaling of the turbulence energy dissipation rate," Phys. Fluids 27, 1048 (1984).

${ }^{7}$ K. R. Sreenivasan, "The energy dissipation in turbulent shear flows," in Symposium on Developments in Fluid Dynamics and Aerospace Engineering, edited by S. M. Deshpande, A. Prabhu, K. R. Sreenivasan, and P. R. Viswanath (Interline, Bangalore, India, 1995), pp. 159-190.

${ }^{8}$ K. R. Sreenivasan, "An update on the energy dissipation rate in isotropic turbulence," Phys. Fluids 10, 528 (1998).

9J. L. Lumley, "Some comments on turbulence," Phys. Fluids A4(2), 203 (1992).

${ }^{10}$ U. Frisch, Turbulent Flows (Cambridge University Press, Cambridge, England, 1995).

${ }^{11}$ Y. Kaneda, T. Ishihara, M. Yokokawa, K. Itakura, and A. Uno, "Energy dissipation rate and energy spectrum in high resolution direct numerical simulations of turbulence in a periodic box," Phys. Fluids 15, L21 (2003).

${ }^{12}$ R. A. Antonia, T. Zhou, and G. Romano, "Small-scale turbulence characteristics of two-dimensional bluff body wakes," J. Fluid Mech. 459, 67 (2002).

${ }^{13}$ R. A. Antonia and B. Pearson, "Effect of initial conditions on the mean energy dissipation rate and the scaling exponent," Phys. Rev. E 62, 8086 (2000).

${ }^{14}$ P. Burattini, P. Lavoie, and R. A. Antonia, "On the normalized turbulent energy dissipation rate," Phys. Fluids 17, 098103 (2005).

${ }^{15} \mathrm{~S}$. Goto and J. C. Vassilicos, "The dissipation rate coefficient of turbulence is not universal and depends on the internal stagnation point structure," Phys. Fluids 21, 035104 (2009).

${ }^{16} \mathrm{G}$. Xu, R. A. Antonia, and S. Rajagopalan, "Scaling of mean temperature dissipation rate," Phys. Fluids 12, 3090 (2000). 
${ }^{17} \mathrm{~T}$. Watanabe and T. Gotoh, "Inertial-range intermittency and accuracy of direct numerical simulation for turbulence and passive scalar turbulence," New J. Phys. 6, 1 (2004).

${ }^{18}$ D. A. Donzis, K. R. Sreenivasan, and P. K. Yeung, "Scalar dissipation rate and dissipative anomaly in isotropic turbulence," J. Fluid Mech. 532, 199 (2005).

${ }^{19}$ B. J. McKeon and J. F. Morrison, "Asymptotic scaling in turbulent pipe flow,” Philos. Trans. R. Soc. London, Ser. A 365, 771 (2007).

${ }^{20}$ Y. Nagano and C. Kim, "A two-equation model for heat transport in wall turbulent shear flows,” ASME J. Heat Transfer 110, 583 (1988).

${ }^{21}$ A. Yoshizawa, "Statistical modelling of passive-scalar diffusion in turbulent shear flows," J. Fluid Mech. 195, 541 (1988).

${ }^{22} \mathrm{~S}$. Hoyas and J. Jiménez, "Reynolds number effects on the Reynolds-stress budgets in turbulent channels," Phys. Fluids 20, 101511 (2008).

${ }^{23}$ J. Kim and R. A. Antonia, "Isotropy of the small scales of turbulence at low Reynolds number,” J. Fluid Mech. 251, 219 (1993).

${ }^{24}$ R. A. Antonia and J. Kim, "A numerical study of local isotropy of turbulence,” Phys. Fluids 6(2), 834 (1994).

${ }^{25}$ H. M. Blackburn, N. N. Mansour, and B. J. Cantwell, "Topology of finescale motions in turbulent channel flow," J. Fluid Mech. 310, 269 (1996).

${ }^{26} \mathrm{H}$. Abe, R. A. Antonia, and H. Kawamura, "Correlation between smallscale velocity and scalar fluctuations in a turbulent channel flow," J. Fluid Mech. 627, 1 (2009).

${ }^{27}$ R. A. Antonia, H. Abe, and H. Kawamura, "Analogy between velocity and scalar fields in a turbulent channel flow," J. Fluid Mech. 628, 241 (2009).

${ }^{28}$ H. Abe, H. Kawamura, and Y. Matsuo, "Surface heat-flux fluctuations in a turbulent channel flow up to $\operatorname{Re}_{\tau}=1020$ with $\operatorname{Pr}=0.025$ and 0.71 ," Int. J. Heat Fluid Flow 25, 404 (2004).

${ }^{29}$ N. Kasagi, Y. Tomita, and A. Kuroda, "Direct numerical simulation of passive scalar field in a turbulent channel flow," ASME J. Heat Transfer 114, 598 (1992).

${ }^{30}$ J. Kim and P. Moin, "Transport of passive scalars in a turbulent channel flow," in Turbulent Shear Flows, edited by J.-C. André, J. Cousteix, F. Durst, B. E. Launder, F. W. Schmidt, and J. H. Whitelaw (Springer-Verlag, Berlin, Germany, 1989), Vol. 6, pp. 85-96.

${ }^{31}$ Z. W. Hu, C. L. Morfey, and N. D. Sandham, "Wall pressure and shear stress spectra from direct numerical simulations of channel flow," AIAA J. 44(7), 1541 (2006).

${ }^{32}$ P. R. Spalart, "Direct simulation of a turbulent boundary layer up to $R_{\theta}=1410$," J. Fluid Mech. 187, 61 (1988).

${ }^{33}$ M. P. Simens, J. Jiménez, S. Hoyas, and Y. Mizuno, “A high-resolution code for turbulent boundary layers," J. Comp. Phys. 228, 4218 (2009).

${ }^{34}$ P. Schlatter, R. Örlü, Q. Li, G. Brethouwer, J. H. M. Fransson, A. V. Johansson, P. H. Alfredsson, and D. S. Henningson, "Turbulent boundary layers up to $R e_{\theta}=2500$ studied through simulation and experiment," Phys. Fluids 21, 051702 (2009).

${ }^{35} \mathrm{G}$. Comte-Bellot, "Contribution àl'étude de la turbulence de conduite," $\mathrm{Ph}$. D. thesis (University of Grenoble, France, 1963) (trans. P. Bradshaw ARC 31609 FM 4102, 1969).

${ }^{36}$ N. Hutchins and I. Marusic, "Evidence of very long meandering features in the logarithmic region of turbulent boundary layers," J. Fluid Mech. 579, 1 (2007).
${ }^{37}$ J. P. Monty and M. S. Chong, "Turbulent channel flow: Comparison of streamwise velocity data from experiments and direct numerical simulation," J. Fluid Mech. 633, 461 (2009).

${ }^{38} \mathrm{P}$. Bradshaw, "The turbulence structure of equilibrium boundary layers," J. Fluid Mech. 29, 625 (1967).

${ }^{39}$ N. Kasagi and Y. Ohtsubo, "Direct numerical simulation of low Prandtl number thermal field in a turbulent channel flow," in Turbulent Shear Flows, edited by F. Durst, R. Friedrich, B. E. Launder, F. W. Schmidt, U. Schumann, and J. H. Whitelaw (Springer, Berlin, Germany, 1993), Vol. 8, pp. 97-119.

${ }^{40}$ H. Kawamura, K. Ohsaka, H. Abe, and K. Yamamoto, "DNS of turbulent heat transfer in channel flow with low to medium-high Prandtl number fluid,” Int. J. Heat Fluid Flow 19, 482 (1998).

${ }^{41}$ H. Kawamura, H. Abe, and Y. Matsuo, "DNS of turbulent heat transfer in channel flow with respect to Reynolds and Prandtl number effects," Int. J. Heat Fluid Flow 20, 196 (1999).

${ }^{42} \mathrm{~F}$. Schwertfirm and M. Manhart, "DNS of passive scalar transport in turbulent channel flow at high Schmidt numbers," Int. J. Heat Fluid Flow 28, 1204 (2007).

${ }^{43}$ M. Kozuka, Y. Seki, and H. Kawamura, "DNS of turbulent heat transfer in a channel flow with a high spatial resolution," Int. J. Heat Fluid Flow 30, 514 (2009).

${ }^{44}$ S. B. Pope, "Consistent modeling of scalars in turbulent flows," Phys. Fluids 26, 404 (1983).

${ }^{45}$ T. Wei and W. W. Willmarth, "Reynolds-number effects on the structure of a turbulent channel flow," J. Fluid Mech. 204, 57 (1989).

${ }^{46}$ B. J. Balakumar and R. J. Adrian, "Large- and very-large-scale motions in channel and boundary-layer flows," Philos. Trans. R. Soc. London $\mathbf{3 6 5}$, 665 (2007).

${ }^{47}$ J. P. Monty, "Developments in smooth wall turbulent duct flows," Ph. D. thesis (University of Melbourne, Australia, 2005).

${ }^{48}$ N. Hutchins, T. B. Nickels, I. Marusic, and M. S. Chong, "Hot-wire spatial resolution issues in wall-bounded turbulence," J. Fluid Mech. 635, 103 (2009).

${ }^{49}$ S. G. Saddoughi and S. V. Veeravalli, "Local isotropy in turbulent boundary layers at high Reynolds number," J. Fluid Mech. 268, 333 (1994).

${ }^{50}$ K. Iwamoto, Y. Suzuki, and N. Kasagi, "Reynolds number effect on wall turbulence: Toward effective feedback control," Int. J. Heat Fluid Flow 23, 678 (2002).

${ }^{51}$ J. Jiménez, and S. Hoyas, "Turbulent fluctuations above the buffer layer of wall-bounded flows,” J. Fluid Mech. 611, 215 (2008).

${ }^{52}$ H. M. Nagib and K. A. Chauhan, "Variations of von Kármán coefficient in canonical flows," Phys. Fluids 20, 101518 (2008).

${ }^{53}$ I. Marusic, B. J. McKeon, P. A. Monkewitz, H. M. Nagib, A. J. Smits, and K. R. Sreenivasan, "Wall-bounded turbulent flows at high Reynolds numbers: Recent advances and key issues," Phys. Fluids 22, 065103 (2010).

${ }^{54}$ J. P. Monty, N. Hutchins, H. C. H. Ng, I. Marusic, and M. S. Chong, "A comparison of turbulent pipe, channel and boundary layer flows," J. Fluid Mech. 632, 431 (2009). 